\title{
IN-VIVO NMR SPECTROSCOPY OF THE BRAIN AT High FIELDS
}

\author{
Rolf Gruetter ${ }^{1,2}$, Pierre-Gilles Henry ${ }^{1}$, Hongxia Lei ${ }^{1}$, \\ Silvia Mangia ', Gülin Öz ${ }^{1}$, Melissa Terpstra ${ }^{1}$, and Ivan Tkac ${ }^{1}$ \\ Departments of Radiologyl and Neuroscience ${ }^{2}$, \\ University of Minnesota, Minneapolis
}

\section{INTRODUCTION}

Increased magnetic fields in principle provide increased sensitivity and specificity. In vivo, however, the increase in magnetic field alone does not automatically result in obvious improvements. Among the factors that are set to impede the improvements in sensitivity for in-vivo NMR spectroscopy are the increased challenges in eliminating the macroscopic inhomogeneities caused by mainly the airtissue interface and increased RF power requirements. Changes in relaxation times may in addition adversely affect the increases in sensitivity, as $T_{1}$ tends to increase and $T_{2}$ tends to decrease with higher magnetic field. In the past 10 years at field strengths of 4 Tesla and higher, we have delineated technical advances that have permitted garnering the advantages of higher field, resulting in substantial gains for ${ }^{1} \mathrm{H}$ and ${ }^{13} \mathrm{C}$ NMR spectroscopy. The improvements can be broadly classified into increased sensitivity, leading to smaller volumes and shorter acquisition times and increased specificity, leading to the detection of many novel compounds. In dynamic ${ }^{13} \mathrm{C}$ NMR it was shown that, in addition to measuring the label incorporation into several positions of many compounds, the time-resolved measurement of isotopomers was possible in the brain in vivo, leading to dynamic isotopomer analysis, a fusion of previously existing techniques. Improvements in sensitivity further advanced the use of localization in ${ }^{13} \mathrm{C}$ NMR spectroscopy, which was critical in detection of brain glycogen metabolism in humans and rodents. Advances in ${ }^{1} \mathrm{H}$ NMR spectroscopy permitted the precise measurement of an array of neurochemicals, ranging from Vitamin $\mathrm{C}$, to glutathione, to glutamine, resulting in an extensive neurochemical profile of different extent that can be measured, e.g., in the unilateral mouse hippocampus, and human substantia nigra. In addition, at high field the simultaneous but separate measurement of $\mathrm{PCr}$ and $\mathrm{Cr}$ was shown to be possible. Sensitivity gains allowed minimization of the detrimental effects of motion by single-scan phase and frequency correction, as well as measurement of the 
neurochemical profile in individuals. In summary, high field ultimately provides NMR spectroscopy with the power to measure highly specific changes in unilateral regions in individual subjects, making it a powerful investigative tool, ideally suited to study pathogenesis and therapy monitoring of disease in humans and animal models, as well as normal brain function. The high specificity of NMR spectroscopy at high fields is likely to result in new insights and improved treatment of human disease.

This chapter first reviews the solutions to some technological challenges at high $B_{0}$, then reviews the methodologies of ${ }^{13} \mathrm{C}$ and ${ }^{1} \mathrm{H}$ NMR spectroscopy at high field, and then briefly reviews some of the biomedical problems uniquely addressed at high field. Current applications that will be reviewed include neurodegenerative diseases, diabetes, and mice.

\section{GENERAL SENSITIVITY CONSIDERATIONS}

It is well known that increasing the static field $B_{0}$ increases the sensitivity of NMR detection [1]. The increase in sensitivity for non-loading samples has been reported as being an exponential function of $B_{0}$, which can be written as

$\mathrm{S} / \mathrm{N} \propto B_{0}{ }^{\beta}$.

From theoretical considerations, it is clear that the signal $S$ increases with $B_{0}{ }^{2}$, hence $\beta$ must be 2 or lower, amounting to an upper limit for the gains in sensitivity achievable due to increases in $B_{0}$ alone. Even when the noise present in the RF coil is the dominant source of noise, its root-mean-square amplitude may increase with frequency $\left(B_{0}\right)$, and it is generally accepted that in this case $\beta=1.75$. For in-vivo spectroscopy, however, the sample is the most dominant source of noise, and thus the noise detection efficiency increases with frequency, and thus the sensitivity increase with $B_{0}$ is closer to linearity with $\beta \sim 1$. Because the sample may not be a completely dominant source of noise, it is plausible that in vivo $\beta$ may lie somewhere between 1 and 1.75 . In practice $\beta$ is probably closer to the former. In this discussion it was assumed that resonance linewidths and relaxation times are very similar between the different field strengths. An increase in resonance linewidth can offset some of the sensitivity gains, as can an increase in longitudinal relaxation time, although both effects influence sensitivity only with the square root of $T_{1}$ or $T_{2}{ }^{*}$. Given that the sensitivity decreases only with $T_{1}^{-1 / 2}$ and that $T_{1}$ appears to increase at most modestly with $B_{0}, T_{1}$ relaxation is unlikely to substantially affect the sensitivity. In contrast, proton $T_{2}$ decreases with $B_{0}$ quite markedly, which will diminish the sensitivity advantage at long echo times [2].

Lastly, increased spectral resolution can result in increased sensitivity due to decreased signal overlap. Although spectral overlap may in general not be a problem for direct-detected ${ }^{13} \mathrm{C}$ NMR spectroscopy, the spectral region of the $\mathrm{C} 2$ of amino acids and the overlap between GABA $\mathrm{C} 2$ and glutamate $\mathrm{C} 4$ stand to gain from increased spectral resolution. 
For ${ }^{1} \mathrm{H}$ NMR spectroscopy, spectral overlap is a much more prevalent problem, and increases in spectral resolution, expressed in ppm, may very well offset defacto increases in intrinsic linewidth, expressed in $\mathrm{Hz}$. For example, in the adult human brain (gray matter) the singlet linewidths are at 1.5 Tesla, typically $\sim 3 \mathrm{~Hz}$ $(0.05 \mathrm{ppm})$, and at 4 Tesla $7 \mathrm{~Hz}(0.04 \mathrm{ppm})$, whereas at $7 \mathrm{~T}$ singlet linewidths are approximately $10 \mathrm{~Hz}(0.03 \mathrm{ppm})$. The novel ability of ${ }^{1} \mathrm{H}$ NMR to resolve $\mathrm{PCr}$ from $\mathrm{Cr}$ at $9.4 \mathrm{~T}$ [3-6] illustrates the ability of increased spectral resolution to help detect new resonances. Furthermore, substantial gains in sensitivity are expected for quantification of signals from more weakly represented metabolites, of which almost all are $J$-coupled. For most resonances, $J$-coupling increasingly approaches the weak coupling limit, in which case the linewidth of a peak in vivo is typically dominated by the homonuclear coupling, as can be illustrated with the example of the apparent Glu H4 triplet at $2.35 \mathrm{ppm}$. Due to the homonuclear $J$-coupling the resonance spreads over an apparent linewidth of about $20 \mathrm{~Hz}$ at almost any field measured from 1.5 Tesla on upwards, and the linewidth is thus dominated by hyperfine splitting of the resonance. Even if the intrinsic linewidth (as measured, e.g., from the singlet resonances) increases by a few $\mathrm{Hz}$ with $B_{0}$, this will have minimal impact on the apparent linewidth of the multiplet. This is illustrated in Figure 1, where the Glu H4 resonance is almost twofold narrower at 7 than at 4 Tesla, which enables separation of the $\mathrm{H} 4$ resonance of glutamate from the $\mathrm{H} 4$ of glutamine at 7 Tesla.

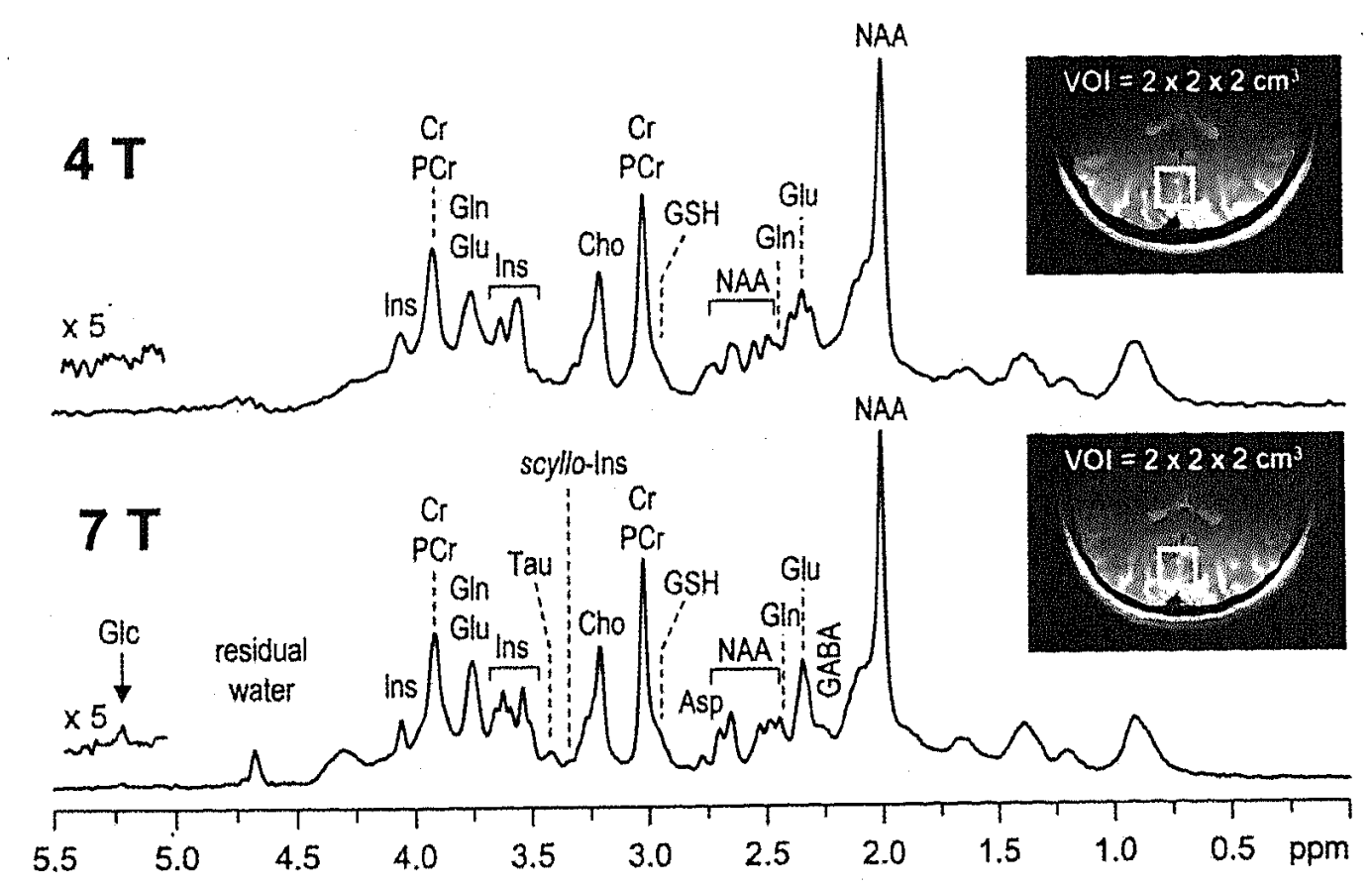

Figure 1. Increased spectral resolution in 'H NMR spectra from the human brain at 7 Tesla (bottom) compared to 4 Tesla (top). Note the apparent and profound reduction in linewidth for the Glu $\mathrm{H} 4$ resonance at $2.35 \mathrm{ppm}$ and the improved resolution even for singlets, as judged from the region separating the $\mathrm{Cho}$ and $\mathrm{Cr}$ resonances. The localization is shown in the inset (MRI, RARE, ETL $=8, \mathrm{TE}=60 \mathrm{~ms}$ ). Spectra were obtained from 8-mL volumes of interest indicated in the same subject and identically processed. 
In practice, it is very difficult to determine the precise value of $\beta$, but it is possible to state the sensitivity gains achieved over previously published results.

\section{RF CONSIDERATIONS}

In principle it may be desirable to have a homogenous RF field covering the entire brain, as it is being widely used for head imaging on conventional MR scanners. When compared to surface coils, the volume coils require increased RF power since the RF power is distributed into a larger volume [1]. Increased RF power implies decreased sensitivity from the reciprocity principle. It is not surprising that volume coils typically are 2-3 times less sensitive than surface coils with optimized dimensions, even when considering the most efficient coil design. With increasing $B_{0}$, effects due to the substantial phase difference of the RF field in the human brain (dielectric resonances) can limit the usefulness of the classic volume coil as a transceiver. In rodents, given the unfavorable small portion of the head occupied by the brain, volume coils may not be the best method to detect the signal. When using surface coils it is advantageous to use adiabatic RF pulses to overcome the problems arising from inhomogenous RF fields, which have been used in a number of studies for NMR spectroscopy [7-15].

With these considerations, and given the unavoidable consequence of inhomogenous RF field at high $B_{0}$, it is expected that multi-array RF coils are likely to be increasingly valuable at high $B_{0}$.

One potential risk to an MR examination is the RF power being absorbed by the tissue converted to heat, which can potentially lead to excessive heating of the body and local tissue, resulting in damage. It is therefore important to limit the RF power administered to the brain, especially for human studies. A simple method to estimate the average specific absorption rate (SAR) is to divide the power dissipated into the organ by the organ volume. However, this calculation is probably an increasingly inaccurate approximation, with the increased spatial inhomogeneity of the RF field at higher frequencies, and other calculations may serve to estimate RF power deposition, such as the following: The current induced in a conducting tissue like the human body is typically dependent on the area of tissue (Faraday's law of induction), which implies that the power deposited increases with the area squared, whereas the volume into which this power is deposited is proportional to the area. These considerations imply that RF power deposition increases with the area of the tissue excited by a given coil. Recent simulations of SAR show that the areas of highest power absorption are at the periphery of the brain, rather than the center [16], probably due to the aforementioned effect. While the perils of so-called hot spots are well established for surface coils, it may also be worth considering the potential increases in local SAR when using whole-body RF coils due to the large organ size.

In general, most of the energy transferred by RF into tissue is converted to heat. While heat bears no specific characteristic related to the frequency of the applied RF, the power absorbed does increase with frequency. These considerations 
make it clear that SAR considerations and application guidelines are largely independent of $B_{0}$. The increased power absorption with frequency thus may simply imply reduced ability to apply the same pulse sequence as at lower field. To minimize the SAR requires that the pulse sequences and decoupling power are adjusted to operate at the minimum power threshold at which sequence performance is still acceptable. For ${ }^{13} \mathrm{C}$ NMR spectroscopy, optimization of RF power is most reliably achieved with an external reference sphere filled with a suitable ${ }^{13} \mathrm{C}$-labeled compound, such as formic acid, placed at the coil center. Highly efficient and accurate methods for adjusting the decoupling power have been described $[17,18]$. In general, the brunt of the SAR is generated by decoupling. For ${ }^{1} \mathrm{H}$ NMR spectroscopy, the RF field (and RF power) can be calibrated on the VOI using the tissue water or fat signal. Typically for ${ }^{1} \mathrm{H}$ NMR spectroscopy, SAR is not of concern unless long $\mathrm{RF}$ pulse trains are applied, such as ${ }^{13} \mathrm{C}$ decoupling or broadband $\mathrm{RF}$ pulses for OVS.

\subsection{Shimming}

The ability to harvest the potential sensitivity gains at high field and the quality of any NMR spectrum stands or falls with the quality of the shimming achieved. This is especially crucial in vivo with increasing $B_{0}$, where the difference between a diamagnetic subject and paramagnetic oxygen in air leads to susceptibilityinduced spatial variations in $B_{0}$ that can be detrimental to the quality of the data obtained. When considering reaping the benefits of high field NMR spectroscopy, issues need to be addressed - namely the choice of shim coils to include in the set of coils provided, and the shim strength that needs to be attained. In this discussion it is important to recognize that the diameter of the shim coils is an important parameter, yet it is not the sole determining factor in defining shim strength. A second point to consider is the scope of the applications one wishes to pursue, as the shim correction currents increase with decreasing head size and they depend strongly on the area within an organ being investigated. First-order shimming is equivalent to adjusting an offset current in the gradient coils, which in general will be sufficient to eliminate first-order $B_{0}$ field variations. For higher-order corrections specialized shim coils will be necessary and the following pertains to their use. In our laboratory we have installed a set of custom-designed shim coils at 9.4 Tesla, capable of generating second-order shim fields up to $0.05 \mathrm{mT} / \mathrm{cm}^{2}$ at a 4 Amp shim current. There are many design issues that need to be considered, but it is likely that the best solution is achieved when additional space outside the gradient coil is available to either accommodate more current within the voltage rail of the shim amplifier or to accommodate extra cooling.

Shim demands increase with decreasing object size, as illustrated by the increased demands on the shim strength when going from rat to mouse brain [19], as shown in Figure 2B. Typically, the quality of a shim current adjustment is based on the measurement of a rather arbitrary parameter - namely the full-width at halfheight. However, this in itself may not be a very good indicator for local adjust- 
ment of the shim coils, and the residual shim fields need to be measured to decide whether the shim process was successful. An algorithm was developed based on FASTMAP [20-23], and its echo-planar-based derivative FASTESTMAP [24], that can adjust with a predefined protocol the first- and second-order shim coils within $30 \mathrm{~s}$ at 4 Tesla and similarly at 7 Tesla in human brain. This method is widely used for high field ${ }^{1} \mathrm{H}$ NMR spectroscopy and can provide rms precision on the order of $0.1 \mathrm{~Hz}$ in the field residual. FASTMAP and closely related variants have been implemented on the platforms of Bruker, Varian, Siemens, SMIS, and Philips.

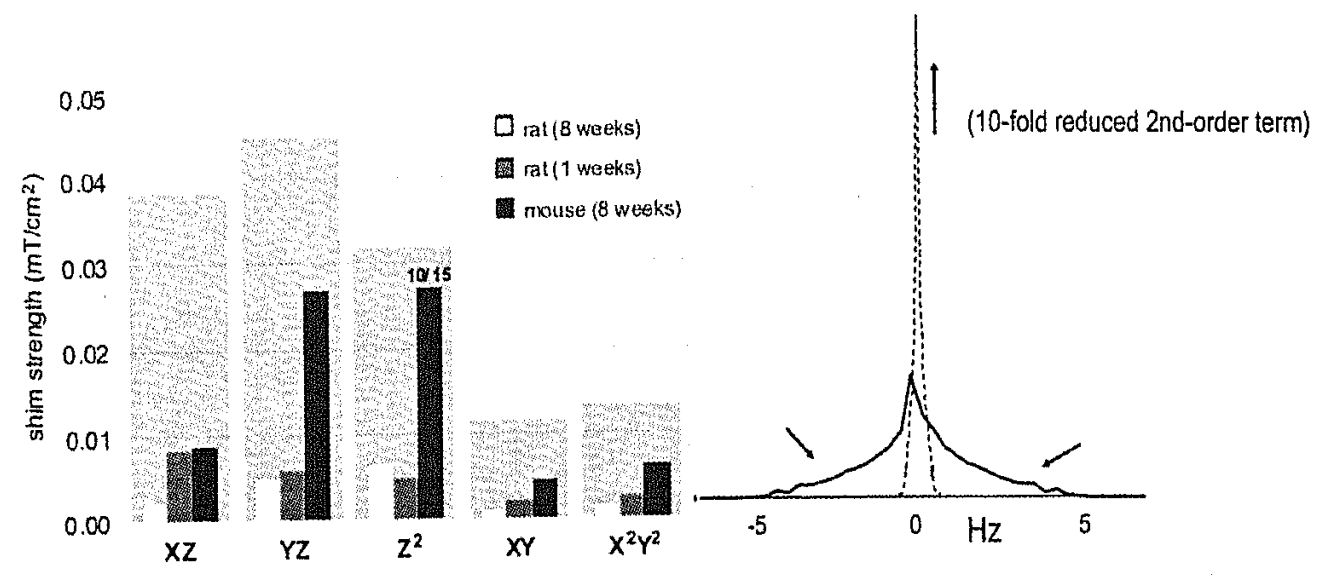

Figure 2. (A) Shim requirements increase with decreasing head size. Average shim strength requirements for mouse, and neonatal and adult rat brain in the unilateral hippocampus at 9.4 Tesla. 10 out of $15 Z_{2}$ shim settings exceeded the maximum available in the mouse brain. Reprinted with permission from PB Barker, DO Hearshen, MD Boska. 2001. Single-voxel proton MRS of the human brain at 1.5T and 3.0T. Magn Reson Med 45:765-769. Copyright ( 2001, John Wiley \& Sons Inc. (B) Effect of second-order shims on lineshape, obtained using a simulation of the frequency distribution in a cubic volume. Shown is the effect of a second-order shim coil (YZ) on the field distribution in a cubic volume. Upon elimination of this term (by shimming), the intensity in the wings is moved underneath the central peak, indicated by the arrows, thereby increasing sensitivity and reducing potential quantification errors.

Even though the effect of $B_{0}$ inhomogeneity on the linewidth of a ${ }^{13} \mathrm{C}$ resonance is reduced by fourfold compared to that of ${ }^{1} \mathrm{H}$, shimming remains an important issue: The low sensitivity of ${ }^{13} \mathrm{C}$ NMR typically precludes measurement of signals from small volumes, leading to comparatively large volumes. The measurement of substantially larger volumes likely results in the need for adjusting the currents in the second-order shim coils (which can be achieved using quantitative shim methods) $[20,22,24]$. The need to adjust second-order shim coils can be appreciated from the fact that the spatial distribution of the $B_{0}$ field of second-order shim coils results in significant signal intensity being distributed in the wings of the resonance (Figure 2B). Such signal distribution can be easily lost in peak integration or even peak fitting at low signal-to-noise ratios, or when using even modest resolution enhancement. The distribution of signal in the wings of a resonance is also likely to affect the quantitative result in ${ }^{1} \mathrm{H}$ NMR spectroscopy, as the precise recovery of such signal intensity even with advanced deconvolution methods is 
difficult in the crowded 'H NMR spectrum. Shimming with second-order shim coils typically has only a modest effect on the full-width at half-maximum of the resonance (Figure 2B). Nonetheless, a significant fraction of the total signal in the wings is shifted under the main resonance, thereby reducing the potential for quantification errors and increasing the sensitivity of the experiment further. These effects are expected to be of increased importance with increased $B_{0}$. In summary, it is likely that adjusting second-order shim coils is necessary to harness the full sensitivity gains at higher field.

With the appropriate design in shim coil hardware and field mapping methods, it was shown that even in the extremely difficult-to-shim mouse brain, highly resolved ${ }^{1} \mathrm{H}$ NMR spectra can be achieved at 9.4 Tesla from distinct regions in the mouse brain (Figure 3), with a spectral quality comparable to that achieved in rat and dog brain $[3-5,25,26]$.
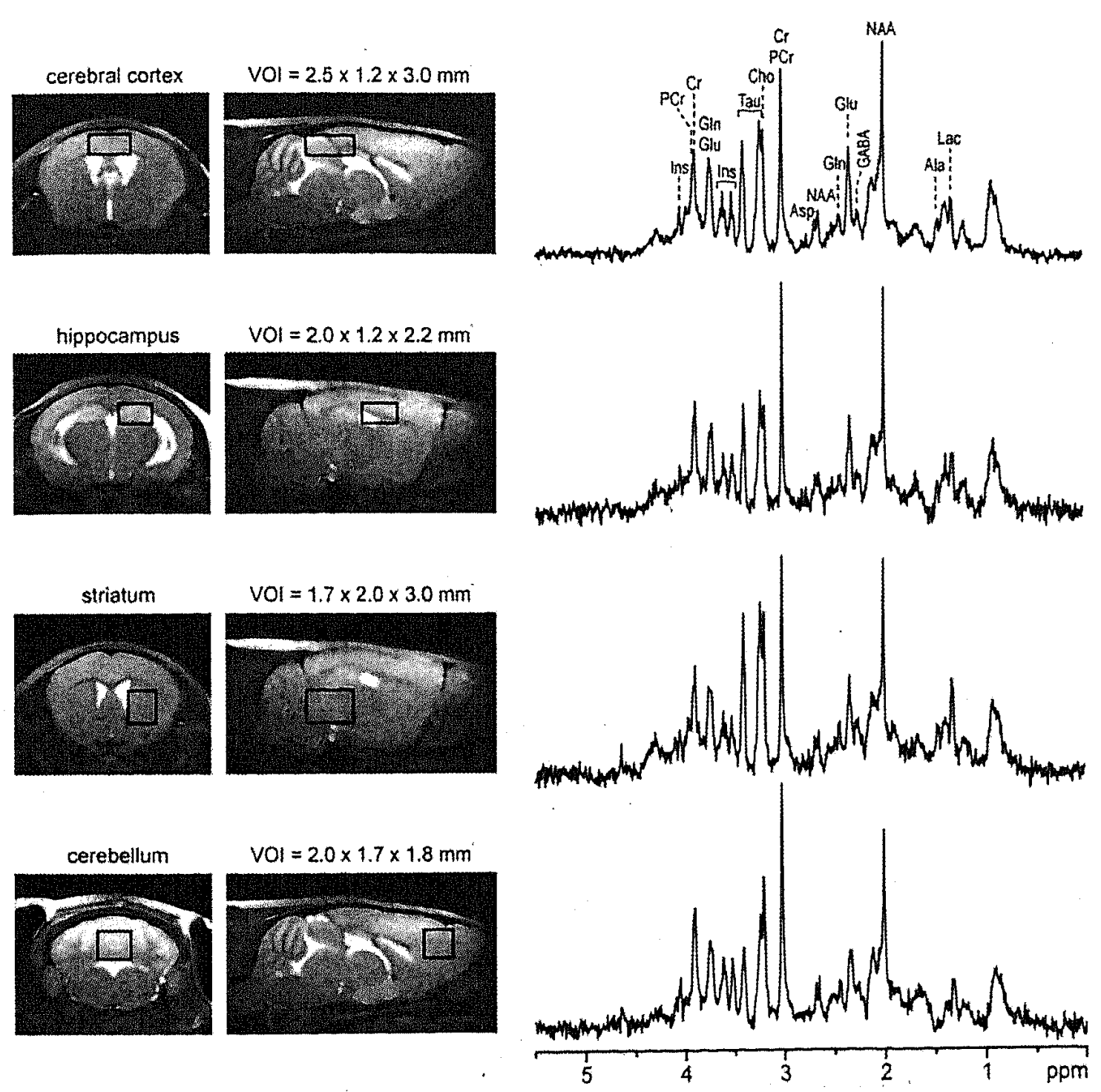

Figure 3. High-resolution in-vivo ${ }^{1} \mathrm{H}$ NMR spectra from distinct anatomic and functional regions in mouse brain. Reprinted with permission from I Tkac, PG Henry, P Andersen, CD Keene, WC Low, R Gruetter. 2004. Highly resolved in vivo ${ }^{1} H$ NMR spectroscopy of the mouse brain at 9.4 T. Magn Reson Med 52:478-484. Copyright @ 2004, John Wiley \& Sons Inc. 


\section{IN-VIVO ${ }^{13} \mathrm{C}$ NMR SPECTROSCOPY}

When considering the sensitivity relative to that of ${ }^{1} \mathrm{H}$ NMR spectroscopy, natural abundance ${ }^{13} \mathrm{C}$ NMR is certainly not expected to compete in the quantitation of a given compound; however, it has proven invaluable in estimating concentrations, which can aid in quantification of the ${ }^{1} \mathrm{H}$ NMR spectrum [27,28]. For example, the difficulty in detecting the natural abundance signal of glutathione (GSH) [29-31] indicates a concentration below $3 \mu \mathrm{mol} / \mathrm{g}$, in agreement with ${ }^{1} \mathrm{H}$ NMR quantification [32], and, similarly, the concentrations of taurine, aspartate, and GABA are expected to be below $2 \mu \mathrm{mol} / \mathrm{g}$ in human brain. The detection of signal from natural abundance compounds in the brain certainly represents a useful complement to in-vivo quantification by ${ }^{1} \mathrm{H}$ NMR spectroscopy $[8,31,33-35]$, albeit of limited practical value.

When a suitable precursor, such as glucose, is administered with enriched ${ }^{13} \mathrm{C}$ label, the label is transferred to molecules in the metabolic pathway. In this manner not only can the sensitivity be increased, but important information on metabolic pathways can also be obtained. In addition to its importance in assessing metabolism in intact brain, ${ }^{13} \mathrm{C}$ NMR spectroscopy has become an important and useful tool in assessing compartmentation of metabolism in brain cells using extracts [36,37].

There are two principal detection methods available - namely direct and indirect detection of ${ }^{13} \mathrm{C}$ label. For indirect detection via ${ }^{1} \mathrm{H}$ resonance coupled to ${ }^{13} \mathrm{C}$, the advantages of high field apply equally well as for regular ${ }^{1} \mathrm{H}$ NMR spectroscopy (see below).

In addition to its low sensitivity, ${ }^{13} \mathrm{C}$ NMR spectroscopy is methodologically more challenging than ${ }^{1} \mathrm{H}$ or even ${ }^{31} \mathrm{P}$ NMR spectroscopy. In contrast to the cell culture and suspension studies and related important work, in vivo ${ }^{13} \mathrm{C}$ NMR spectroscopy of intact organs faces a number of problems that make its application more challenging. Complete three-dimensional localization seems necessary in the brain to eliminate the intense triacylglycerol resonances from the scalp and other signals outside the brain, which adds to the challenges of in-vivo ${ }^{13} \mathrm{C} N M R$. The application of ${ }^{13} \mathrm{C}$ NMR spectroscopy to the brain faces in-vivo some challenges on the technical level that are unique to the ${ }^{13} \mathrm{C}$ nucleus and compounded at high magnetic fields. This section first examines the reverberations of the requirement to decouple the spectrum during acquisition, and then discusses the localization requirements. A more extensive discussion of these issues and technical questions can be found in [30].

\subsection{Decoupling: RF Power Considerations}

Many ${ }^{13} \mathrm{C}$ nuclei are directly bonded to protons. The resulting magnetic coupling between the ${ }^{13} \mathrm{C}$ nucleus and the ${ }^{1} \mathrm{H}$ nucleus results in a splitting of the ${ }^{13} \mathrm{C}$ resonance into multiplets separated by $J_{\mathrm{CH}} \mathrm{Hz}$. The process of decoupling collapses the multiplets due to heteronuclear coupling into singlets, thereby simplifying the spectra and effectively increasing the sensitivity. Optimization of sensitivity is 
critical for successful in-vivo ${ }^{13} \mathrm{C}$ NMR spectroscopy. To maximize the signal-tonoise ratio and spectral resolution in ${ }^{13} \mathrm{C}$ NMR spectra, ${ }^{1} \mathrm{H}$ decoupling is generally applied during data acquisition, resulting in a simplified spectral pattern. The application of rather intense RF power during acquisition requires that the RF coil be capable of receiving the ${ }^{13} \mathrm{C}$ NMR signal while transmitting ${ }^{1} \mathrm{H}$ RF power. This places several requirements on the spectrometer console, RF coils, and RF filters.

The need for decoupling results in two requirements for RF coils: First, the ${ }^{13} \mathrm{C}$ coil (operating at a frequency with a wavelength approximately 4 times that of the ${ }^{1} \mathrm{H}$ frequency) should not interfere with the RF profile of the ${ }^{1} \mathrm{H}$ coils. Second, the two RF circuits should be sufficiently isolated electrically. For a further discussion of RF coil design for ${ }^{13} \mathrm{C}$ NMR spectroscopy at high fields, see [30].

Most ${ }^{13} \mathrm{C}$ NMR applications have relied on WALTZ decoupling of the protons [38]. Broadband adiabatic ${ }^{13} \mathrm{C}$ decoupling was achieved at 9.4 Tesla using such cycles at a moderate peak $\gamma B_{1} / 2 \pi$ of $\sim 1 \mathrm{kHz}$ [39] and at 7 Tesla [40].

\subsection{Localization Methods for ${ }^{13} \mathrm{C}$ NMR Spectroscopy}

The full chemical shift range of most biologically interesting ${ }^{13} \mathrm{C}$ resonances is approximately 160-200 ppm wide. Even when considering the fourfold lower gyromagnetic ratio than ${ }^{1} \mathrm{H}$, this is a range that (in $\mathrm{Hz}$ ) exceeds the range for ${ }^{1} \mathrm{H}$ resonances of main biological interest ( $7 \mathrm{ppm}$ ) by at least a factor of six (Table 1). Thus, chemical shift displacement error problems are important factors to consider when wishing to localize ${ }^{13} \mathrm{C}$ NMR signals.

Table 1. Chemical Shift Ranges for ${ }^{13} \mathrm{C}$ MRS per Tesla (Expressed in $\mathrm{Hz} / \mathrm{T}$ ), Including the Range of the Corresponding Coupled ${ }^{1} \mathrm{H}$ Resonances

\begin{tabular}{lccc}
\hline Compound class & $\begin{array}{c}\text { Chemical shift } \\
\text { range }(\mathrm{ppm})\end{array}$ & $\begin{array}{c}{ }^{13} \mathrm{C} \text { frequency } \\
\text { range (Hz/T) }\end{array}$ & $\begin{array}{c}{ }^{1} \mathrm{H} \text { frequency } \\
\text { range (Hz/T) }\end{array}$ \\
\hline Glycogen/glucose (all) & $101-61$ & 430 & 94 \\
Myo-inositol (all) & $76-72$ & 43 & 34 \\
Amino acids $\left(\mathrm{CH}_{\mathrm{n}}\right)$ & $56-22$ & 363 & 85 \\
Amino acids $(\mathrm{CH})$ & $56-53$ & 32 & 20 \\
Lipids $\left(\mathrm{CH}_{\mathrm{n}}\right)$ & $131-14$ & 1248 & 196 \\
\hline
\end{tabular}

Spatial localization does not depend on chemical shift when using the onedimensional surface-spoiling gradient or spectroscopic imaging $[41,42]$, which is an elegant technique to solve the chemical shift displacement problem when many transients can be acquired [43]. Typically the rather high number of averages acquired for in-vivo ${ }^{13} \mathrm{C}$ NMR spectroscopy bodes well with the use of chemical shift imaging. However, the low spatial resolution may require dedicated solutions to 
minimize Gibbs ringing from superficial fat signals, and several approaches have been described $[44,45]$. In the following we will concentrate on the various methods for full three-dimensional localization of ${ }^{13} \mathrm{C}$ NMR signals using gradientbased methods.

The use of direct localization of ${ }^{13} \mathrm{C}$ is challenging because the large chemical shift requirement leads in general to increased demand on the RF power through the need for an increased RF bandwidth. In some cases, even when the demands on the RF bandwidth can be met, the required gradient strength may very well be limiting, because of the lower gyromagnetic ratio $\gamma$. Therefore, for practical reasons, direct localization is typically limited to a given spectral range (Table 1), e.g., the direct measurement of brain glucose $\mathrm{Cl}$ in the human brain [9]. In addition, direct localized ${ }^{13} \mathrm{C}$ NMR spectroscopy was used for the first detection of the natural abundance signals of small molecules, as illustrated with myo-inositol [8].

\subsubsection{Localization on ${ }^{\prime} H$ Magnetization: Polarization Transfer}

When using ${ }^{13} \mathrm{C}$ longitudinal magnetization for localization, signal enhancements are commonly achieved using the nuclear Overhauser effect (NOE), which can theoretically provide up to a threefold enhancement of ${ }^{13} \mathrm{C}$ signals. However, for resonances with sufficiently long $T_{2}$, such as those of most brain metabolites as judged from their narrow linewidths of a few $\mathrm{Hz}$ [22], it is feasible to use polarization transfer to recover the maximal sensitivity gain of a fourfold enhancement and to localize on the ${ }^{1} \mathrm{H}$ magnetization, thereby greatly reducing the chemical shift displacement error due to the much smaller chemical shift range (Table 1). Heteronuclear polarization transfer combined with localization on the proton magnetization was shown to minimize the chemical shift displacement error in ${ }^{13} \mathrm{C}$ MRS of the human brain to a level beyond concern even at 4 Tesla [31]. To minimize the number of pulses needed for generating the in-phase ${ }^{13} \mathrm{C}$ signal enhancement and to minimize phase distortions in the spectrum, distortionless enhanced polarization transfer, DEPT [46], was used. The high sensitivity of polarization transfer pulse sequences to variations in $B_{1}$ can be ameliorated for the ${ }^{13} \mathrm{C}$ pulses when incorporating adiabatic pulses into the pulsed polarization transfer sequence $[11,15,47]$. Most polarization methods cannot provide full enhancement over the entire range of $J$-coupling constants, ${ }^{1} \mathrm{H}$ spin multiplicities, and chemical shift range. Nonetheless, a high sensitivity was achieved and rich spectral information were obtained at 4 Tesla in human brain and at 9.4 Tesla in rat brain (Figure 4).

\subsubsection{Three-Dimensional Localization of the ${ }^{13}$ C NMR Signals of Glycogen}

Clearly, the chemical shift displacement error can be minimized by using ${ }^{1} \mathrm{H}$ magnetization for localizing the ${ }^{13} \mathrm{C}$ NMR signals in conjunction with polarization transfer. However, methods such as those discussed in the previous paragraph are not applicable in situations where $T_{2}$ is very short. For some compounds such as glycogen, it is nearly impossible to realize the full sensitivity gains of the polarization transfer technique. While the SINEPT technique [49] relies on minimizing $T_{2}$ losses by performing polarization transfer on-resonance without refocusing the $J$ 
evolution, the method remains in principle susceptible to changes in $T_{2}$, which have been reported to occur depending on the glycogen molecule size and temperature [50]. Unfortunately, the potential fourfold signal gain is reduced by the short $T_{2}$ of ${ }^{1} \mathrm{H}$ and ${ }^{13} \mathrm{C}$ signals - on the order of $5 \mathrm{~ms}$ [50,51]. Given the uncertainty of potential changes in transverse relaxation times [50], a robust method relying on outervolume suppression applied along the six slices parallel to the volume-of-interest was developed. In the brain such localization was crucial to minimize potential contamination of the detected signal with signals from extraneous muscle, especially in the rat $[12,14,52]$.

Glu C4
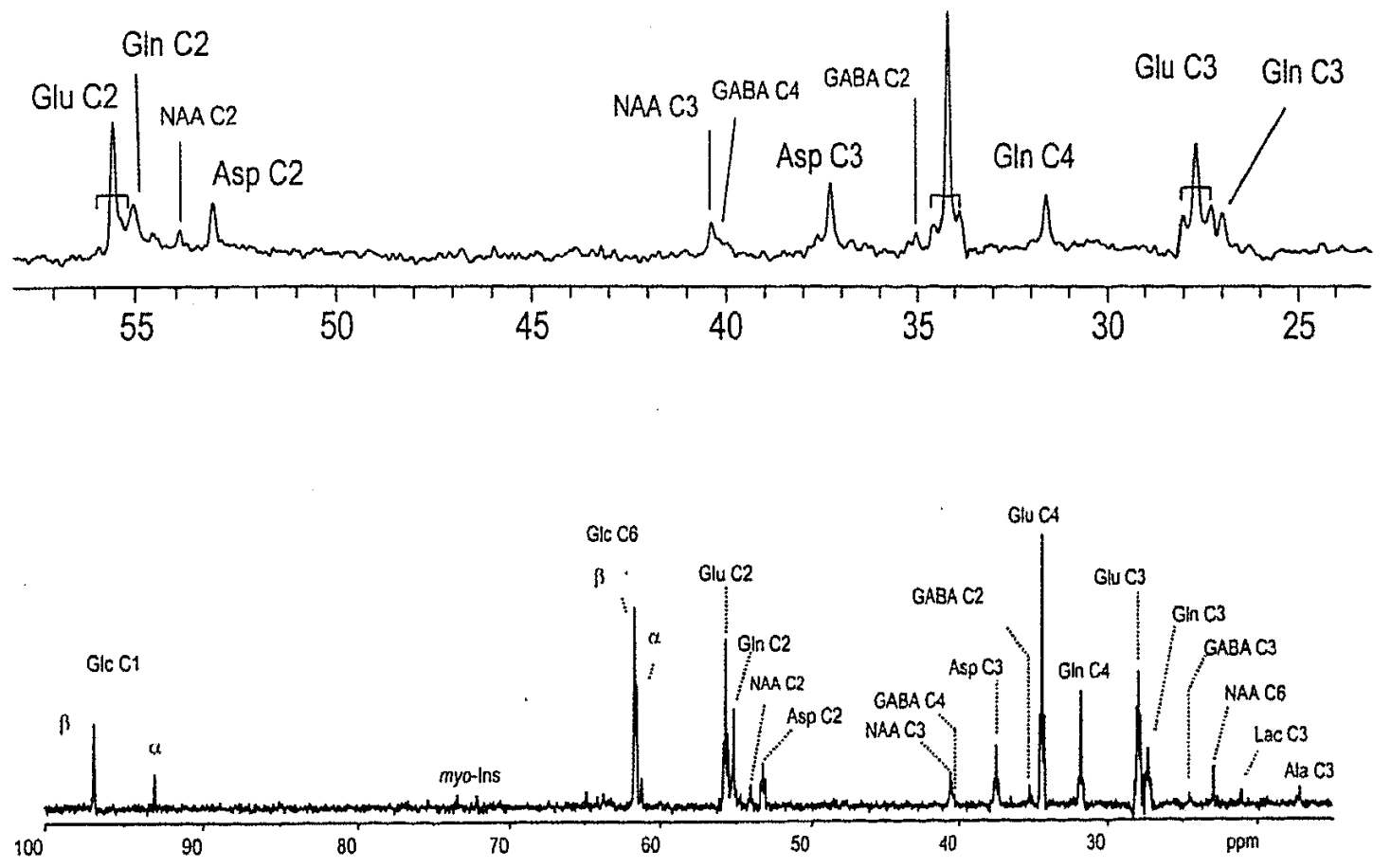

Figure 4. Examples of direct-detected ${ }^{13} \mathrm{C}$ NMR spectroscopy from the brain. (A) ${ }^{13} \mathrm{C}$ NMR detection of label incorporation into mostly cytosolic amino acids at $4 \mathrm{~T}$. Reprinted with permission from $\mathrm{R}$ Gruetter, ER Seaquist, K Ugurbil. 2001. A mathematical model of compartmentalized neurotransmitter metabolism in the human brain. Am J Physiol 281:E100-112. Copyright (C) 2001, American Physiological Society. Shown is a representative spectrum obtained from a $45-\mathrm{ml}$ volume in the human visual cortex during an infusion of $67 \%$-enriched $\left[1-{ }^{13} \mathrm{C}\right]$ glucose. In addition, resonances resulting from homonuclear ${ }^{13} \mathrm{C}-{ }^{13} \mathrm{C}$ coupling were readily detected at the positions of all glutamate resonances (indicated by brackets). Processing consisted of a mild Lorentz-Gauss apodization $(3 \mathrm{~Hz})$ and the spectrum is shown without baseline correction. (B) In-vivo ${ }^{13} \mathrm{C}$ NMR spectra

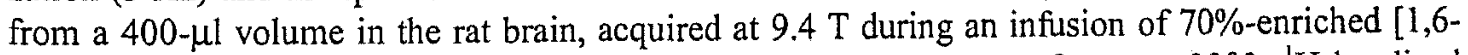
${ }^{13} \mathrm{C} 2$ ]glucose. Reprinted with permission from PG Henry, I Tkac, R Gruetter. 2003. 'H-localized broadband 13C NMR spectroscopy of the rat brain in vivo at 9.4 T. Magn Reson Med 50:684-692. Copyright (C) 2003, John Wiley \& Sons Inc. Processing consisted of zero-filling, $2 \mathrm{~Hz}$ Lorentzianto-Gaussian resolution enhancement and fast Fourier transform. No baseline correction was applied. Note the complete absence of lipid signals over the entire spectral range. Resonance assignments are as follows: Glu C2 at $55.6 \mathrm{ppm}$, Gln C2 at $55.0 \mathrm{ppm}, \mathrm{NAA} C 2$ at $54.0 \mathrm{ppm}, \mathrm{Asp} C 2$ at $53.7 \mathrm{ppm}$, NAA C3 at $40.5 \mathrm{ppm}$, GABA C4 at $40.45 \mathrm{ppm}$, Asp C3 at $37.6 \mathrm{ppm}$, GABA C2 at $35.3 \mathrm{ppm}$, Glu $\mathrm{C} 4$ at $34.2 \mathrm{ppm}$, Gln $\mathrm{C} 4$ at $31.7 \mathrm{ppm}$, Glu $\mathrm{C} 3$ at $28.0 \mathrm{ppm}$, Gln $\mathrm{C} 3$ at $27.7 \mathrm{ppm}$. 


\section{IN-VIVO ${ }^{1}$ H NMR SPECTROSCOPY}

In contrast to ${ }^{13} \mathrm{C}$ NMR spectroscopy, spectral overlap is a much more pronounced problem with ${ }^{1} \mathrm{H}$ NMR spectroscopy, and because the ${ }^{1} \mathrm{H}$ nucleus is much more susceptible to variations in $B_{0}$, shimming (see above) is a critical component in accomplishing the potential gains of higher $B_{0}$. This section deals with the advantages achievable once the problem of shimming can be solved satisfactorily.

\subsection{A Word on Localization Methods}

Despite its inherent disadvantages for in-vivo ${ }^{1} \mathrm{H}$ NMR spectroscopy (sensitivity to motion, dynamic range problems when selecting small volumes) ISIS has been successfully used for spatial localization of ${ }^{1} \mathrm{H}$ NMR in vivo in conjunction with outer volume suppression and mostly with surface coil detection [53].

When using localization based on the more popular stimulated echo (STEAM), the echo time can be minimized to 4-6 ms for human brain and 1-2 ms in rodent brain, effectively minimizing potential $T_{2}$ losses and $J$ evolution effects $[19,54,55]$. However, the method's big disadvantage is the up to twofold sacrifice in sensitivity, which can translate into an up to $26 \%$ increased voxel dimension or up to fourfold increased measuring time at equal size of the volume of interest. It may seem unacceptable to sacrifice the sensitivity by twofold while buying a higher magnetic field system. However, sensitivity increases supralinearly with increasing magnetic field.

The sensitivity disadvantage of STEAM is remedied by using the PRESS or double-echo method, which is highly sensitive to those spatial variations in $B_{1}$ that are increasingly prevalent at high frequencies. Furthermore, considering traditional (nonadiabatic) RF pulses, the peak RF power has to increase by more than fourfold if one wishes to retain the same chemical shift displacement error as for STEAM. Lastly, for a given RF power and gradient slew rate, the echo time (TE) can be substantially longer.

Some of the aforementioned problems inherent with PRESS can be ameliorated when using adiabatic RF pulses. For example, using offset-independent adiabatic pulses [56], inversion pulses with almost arbitrary bandwidths can be generated for a given peak RF amplitude. This is done, however, at the cost of increased $\mathrm{RF}$ pulse duration, as the speed of the adiabatic sweep is reduced. In addition, adiabatic $180^{\circ}$ refocusing pulses do not have good slice profiles, unless when applying pulses of the hyperbolic secant type in pairs, which further increases the echo time. One method, LASER [57], takes advantage of adiabatic single-shot localization. LASER addresses the issue of increased echo time by measuring in Carr-Purcell-Meiboom-Gill mode, where the transverse relaxation is increasingly replaced by $T_{1 p}$ relaxation, which in vivo tends to be longer, thereby minimizing these losses.

Chemical shift imaging [43] will obviously not suffer from chemical shift displacement errors in the dimensions the phase encode gradients are applied. One drawback of the method is that longer acquisition times are necessary to collect the entire data set and that the sensitivity is lower than compared to single-voxel acqui- 
sition. Thus, for those biomedical problems, where the region of interest is already known to the investigator or where spatial distribution of the defect is not important, chemical shift imaging may not be the first choice of method. Another issue stems from the fact that the intense lipid signals typically are associated with rather thin skin layers at the surface and the lower resolution of the chemical shift imaging data set results in Gibbs' ringing that may be motion-sensitive $[44,45]$, which must be dealt with [58]. Lastly, effects of motion are not as easily corrected as for single-voxel spectroscopy, which may limit the use of CSI, especially in pediatric applications [59]. At very high magnetic fields, the spatial resolution achievable due to the higher sensitivity may result in a number of phase-encode steps that lead to prohibitively long acquisition times, hence some form of field-of-view restriction or parallel imaging may be necessary.

Given the undisputed sensitivity advantage of PRESS over STEAM, it may seem surprising to see that many pioneering studies at very high field have successfully used STEAM. There are several reasons that make the choice of STEAM quite attractive, all rooted in the use of essentially three $90^{\circ}$ slice-selective RF pulses: A well-defined slice profile is easier accomplished with a $90^{\circ}$ flip angle, with lower peak RF power and lower average RF power than for $180^{\circ}$ pulses. The use of asymmetric RF pulses permits minimization of the effective echo time to $1 \mathrm{~ms}$ in rodent scanners [25], and to a few $\mathrm{ms}$ in whole-body scanners [54], effectively eliminating the effects of $T_{2}$ relaxation and $J$ evolution to spectral appearance, thereby greatly facilitating quantification. Because the sequence relies on detection of a stimulated echo generated by all three $90^{\circ}$ pulses, fewer stimulated echoes need to be dephased than in PRESS and spin echoes are easier to dephase in the TM period. Lastly, the sequence is inherently less sensitive to variations in $B_{1}$, a critical issue when considering the spatially inhomogenous RF field typically encountered in vivo at very high magnetic fields.

\subsection{Water Suppression}

It has been noted that in general the ${ }^{1} \mathrm{H}$ linewidths (in ppm) decrease with magnetic field strength in the adult brain, which makes it clear that the spectral resolution increases with magnetic field. This is particularly important for the most intense singlet resonance, namely water, as it implies improved water suppression, as was illustrated with the detection of the glucose signal at $5.23 \mathrm{ppm}[34,60]$. The sensitivity gains obtained by better and more reliable water suppression are more difficult to quantify, but they can be appreciated when considering, for example, that fewer spurious water signals from outside the VOI may confound the spectral region of interest, that a flatter baseline will yield better confidence intervals for the measurements. 


\subsection{Spectral Editing: Improved Pulse Selectivity at High Field}

The spectral overlap problem inherent in 'H NMR spectroscopy can be circumvented using spectral editing, which typically exploits the fact that in most metabolites the resonances in a given spin system are magnetically coupled. By specifically manipulating the coherence, the signal of interest can be selectively filtered from the overall background, and it is thus feasible to extract unresolved resonances from underneath much more intense resonances. Examples where this was achieved include GABA, GSH, and lactate [61-63]. Provided the coupling spin system is uniquely excitable, these editing methods are a very powerful tool for the measurement of obscured spin systems. Unfortunately, for one of the most interesting compounds, namely GABA, this uniqueness of spin system does not apply, due to overlap with putative lysine resonances from cytosolic proteins [64]. The traditional editing of the GABA resonance at $3.0 \mathrm{ppm}$ is hampered by the simultaneous excitation of a very similar macromolecule coherence at $1.7 \mathrm{ppm}$ (relative to that of GABA at $1.9 \mathrm{ppm}$ ) and alternative editing methods such as editing for the GABA at $2.3 \mathrm{ppm}$ may prove advantageous [65]. Another type of editing that is applied to ${ }^{1} \mathrm{H}$ spectroscopy is the editing of ${ }^{1} \mathrm{H}$ resonances with magnetic couplings to ${ }^{13} \mathrm{C}$ nuclei, which are at low field obviously hampered by the limited resolution of the ${ }^{1} \mathrm{H}$ spectrum. However, at higher fields such as 9.4 Tesla an increased number of resonances can be measured.

Lastly, it is also possible to edit for coherences using total correlation spectroscopy. Using isotropic mixing, such coherence transfer can be used to recover resonance intensity in a spectral region where signal had previously been eliminated, e.g., the water spectral region [66].

In all these experiments, it is important to recognize that the spectral pattern of the resonance for which the editing is performed in vivo should be very similar to that predicted for the isolated compound as measured under the same experimental conditions (e.g., ionic strength, temperature and $\mathrm{pH}$ ) as illustrated for the case of GSH (Figure 5). In addition, editing experiments are intrinsically less sensitive than measurements done directly on the resonance at short echo times (see below), for two reasons - namely the signal loss due to the longer echo time TE, and the potential signal loss from the editing process (in a resonance coupling to $\mathrm{CH}_{2}$ (e.g., $\mathrm{AX}_{2}$ spin system) the centerline does not evolve with $J$ and is thus filtered away, leading to at least a twofold reduction in sensitivity). Finally, for many editing experiments, but especially for GABA, changes in $B_{0}$ during the experiment can alter the editing efficiency or selectivity, which can result in altered amplitudes and/or contamination, for example, by co-edited macromolecules [67].

\subsection{Spectral resolution: The Neurochemical Profile}

Provided sufficient spectral resolution is present, as has been shown for numerous compounds at high $B_{0}[5,19,54,59]$, it is conceivable that for many compounds spectral editing may not be necessary routinely with increasing sensitivity. As pointed out earlier, the spectral resolution increases in ${ }^{1} \mathrm{H}$ spectra with high 
magnetic field, especially for $J$-coupled resonances when the macroscopic $B_{0}$ inhomogeneities are minimized by shimming. Despite the increased spectral resolution obviously present for coupled ${ }^{1} \mathrm{H}$ spin systems, even at the highest magnetic field applicable in vivo, substantial spectral overlap remains, unless some form of spectral editing (see above) is used. Hence direct peak integration or simple fitting are likely prone to systematic errors.

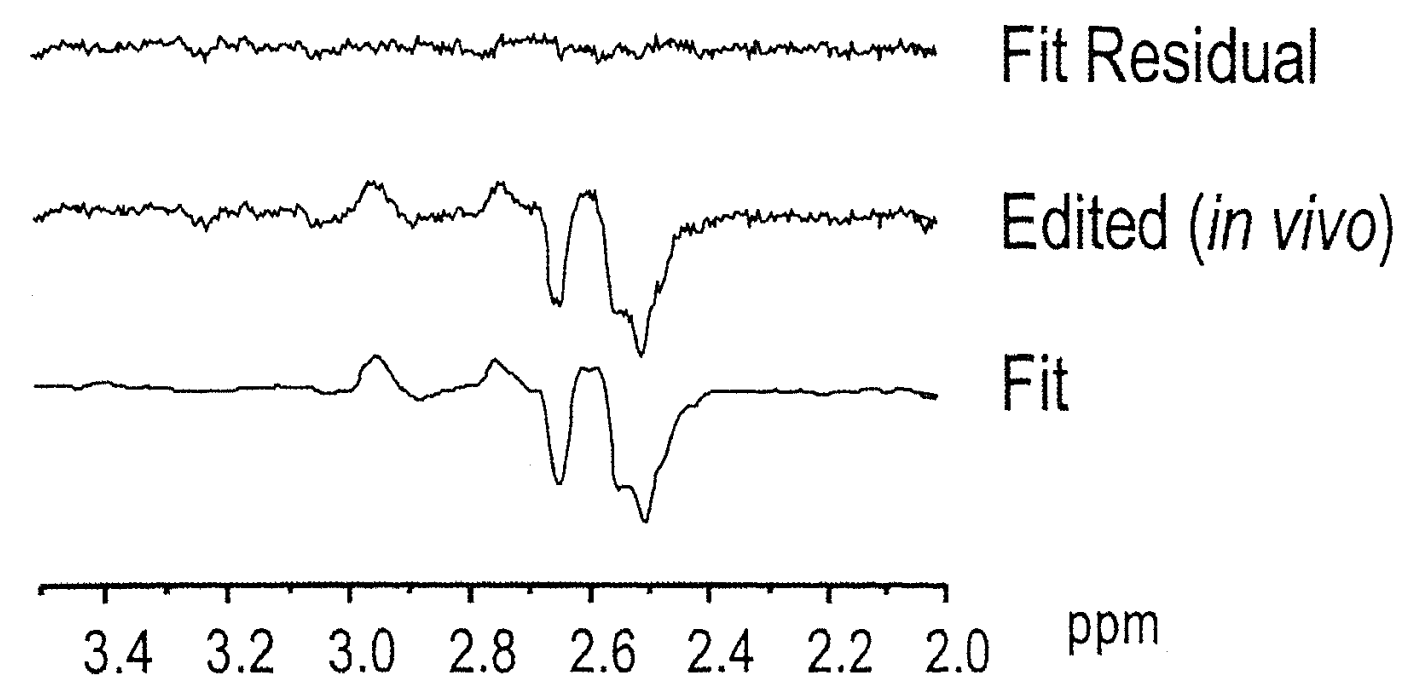

Figure 5. Editing of the spin system of glutathione (GSH) using MEGA-PRESS editing $[67,68]$ at 4 Tesla. The GSH resonance at $2.95 \mathrm{ppm}$ is co-edited with the aspartyl resonance of NAA at $2.6 \mathrm{ppm}$. The in-vivo spectrum (middle) is almost perfectly matched by the LCModel fit of the respective resonances measured in phantom, as indicated by the fit residual (top trace).

At short echo times, approaches that mimic the in-vivo spectral pattern and that incorporate prior knowledge become increasingly powerful, such as TDFDfit [69], LCModel [5,70], MRUI [71,72], etc. These methods are of increasing importance with decreasing $B_{0}$, as overlap increases. Conversely, the increased spectral dispersion at higher field and increased sensitivity propel the use of short-echo ${ }^{1} \mathrm{H}$ NMR spectroscopy for measurements in individual patients. For example, incremental changes of various metabolites, such as glutamate and glutamine, can be assessed longitudinally in patients to follow diseases progression and response to treatment [59]. Such noninvasive assessment of pathology over time is expected to eventually aid inpatient management. Sensitivity and reliability are best assessed from the average Cramer-Rao lower bounds, which assess the reliability of the fitted amplitude, as well as from the covariance matrix.

In the determination of metabolite signals, a faithrully determined baseline may prove critical and essential. Baseline determination in vivo is difficult due to the overlap of resonances in the short-echo ${ }^{1} \mathrm{H}$ spectrum. In addition, a signal background associated with cytosolic proteins (macromolecule background) complicates the shape of the baseline. Fortunately, the $T_{1}$ of these macromolecule reso- 
nances is much shorter than that of most metabolites, which allows the separate measurement of the macromolecule signals in so-called metabolite-nulled spectra [64]. The such-measured complex signal from macromolecules can be incorporated into the basis set of, e.g., LCModel, as reported by Pfeuffer et al. [4,5]. This approach of incorporating the short $T_{1}$ background signals (macromolecules) is increasingly being used for in-vivo ${ }^{1} \mathrm{H}$ NMR spectroscopy of the brain $[4,32,73-75]$. The information content and deconvolution of the in-vivo spectrum is shown in Figure 6 for human brain at 4 Tesla and for rat brain at 9.4 Tesla.

\section{INSIGHTS FROM HIGH FIELD NMR SPECTROSCOPY STUDIES OF THE BRAIN}

In-vivo spectroscopy, especially at high fields, provides the investigator with a unique tool. One strength of the method lies in the deterministic nature of the NMR signal: Not only is it in general known at what chemical shift to expect a signal for a given compound, the spectral features, which can be quite complex, can be faithfully reproduced using either model solution and/or simulations. Additionally, the lineshape is subject to certain constraints of similarity between the different metabolites. Finally, some of the concentrations expected should conform to what is reasonably expected from the underlying neurochemistry. These characteristics can serve to allow a faithful measurement of metabolites in vivo. On one hand, the concurrent quantitative measurement by ${ }^{1} \mathrm{H}$ NMR spectroscopy of many important neurochemicals constituting the neurochemical profile allows for noninvasive insight into the neurochemical consequences of many diseases in human subjects, thereby providing an important translational link. On the other hand, localized NMR spectroscopy of other stable isotopes provides a window on in-vivo brain metabolism, with a unique chemical specificity and diversity of potential measurements not possible by other methods. The metabolism of stable isotopes can be followed noninvasively using NMR, although administration of the "tracer" requires a high isotopic enrichment, for ${ }^{13} \mathrm{C}$ typically above $50 \%$ in the precursor pool. Because NMR spectroscopy can be used to detect label in different molecules and different chemical positions, it offers the attractive possibility to follow the metabolism of the precursor, labeled at one or more specific positions. Applications are predominantly focused on nuclei where a stable isotope is present at low natural abundance - examples include ${ }^{2} \mathrm{H},{ }^{15} \mathrm{~N},{ }^{19} \mathrm{~F}$, and ${ }^{13} \mathrm{C}[76]$.

Of course, sensitivity for NMR is low when considering the concentrations that are detectable, and the relative sensitivity of ${ }^{13} \mathrm{C}$ NMR is even lower. Nonetheless, despite the sensitivity disadvantage, as shall be discussed in the second half of this review, high field NMR spectroscopy can provide unique insights into brain metabolism. Many consider a low sensitivity and thus low spatial resolution a significant disadvantage. However, it is our belief that the spatial resolution that can be achieved combined with the high specificity of the method allows one to uniquely address a significant number of important biomedical problems/questions. 
Human brain, occipital gray matter $-4 \mathrm{~T}$

Rat brain, hippocampus - $9.4 \mathrm{~T}$
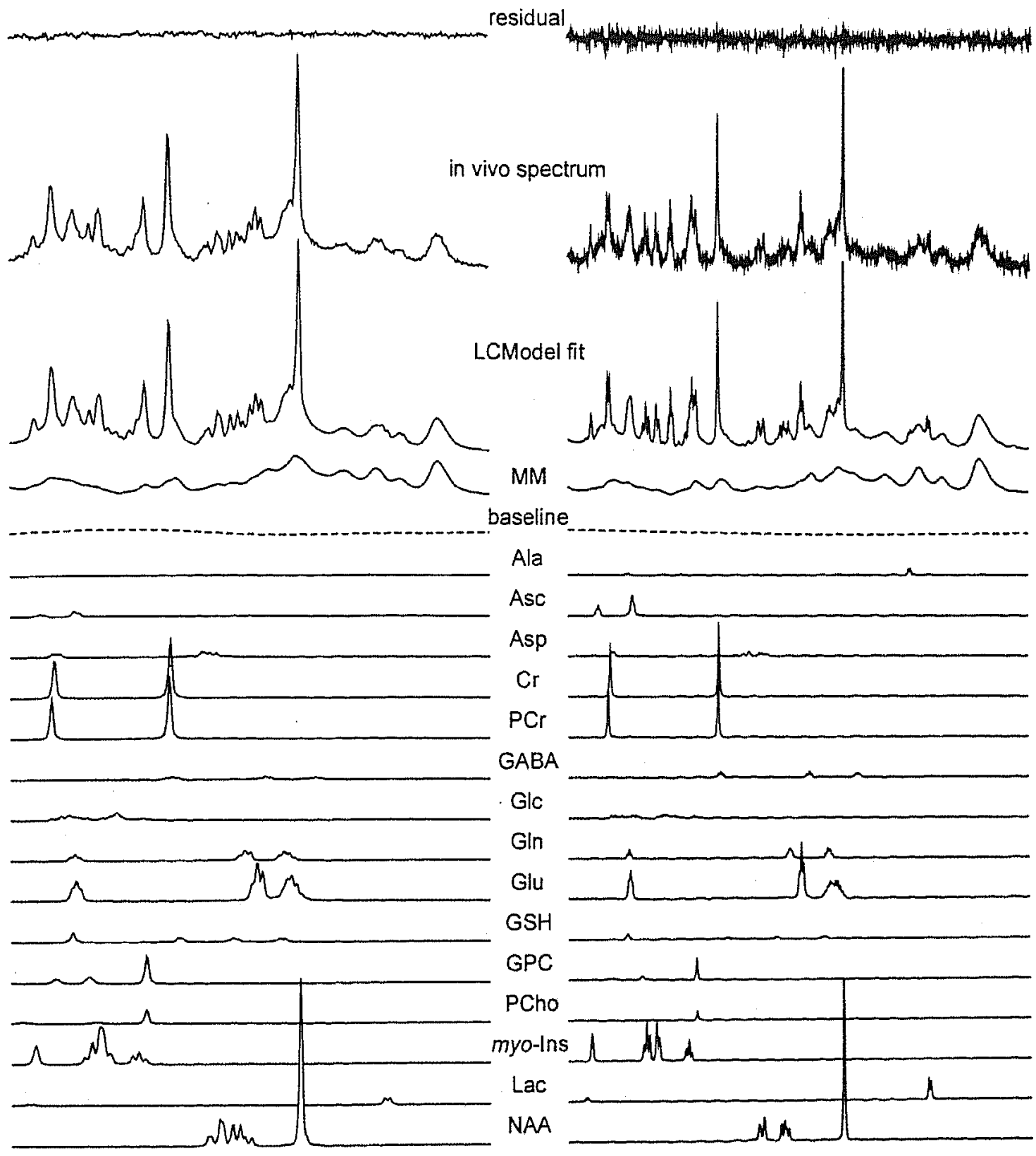

GABA

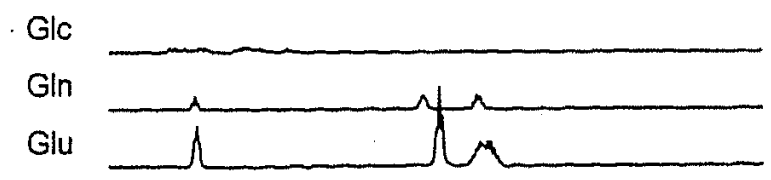

GSH

GPC

PCho myo-Ins

Lac

NAA
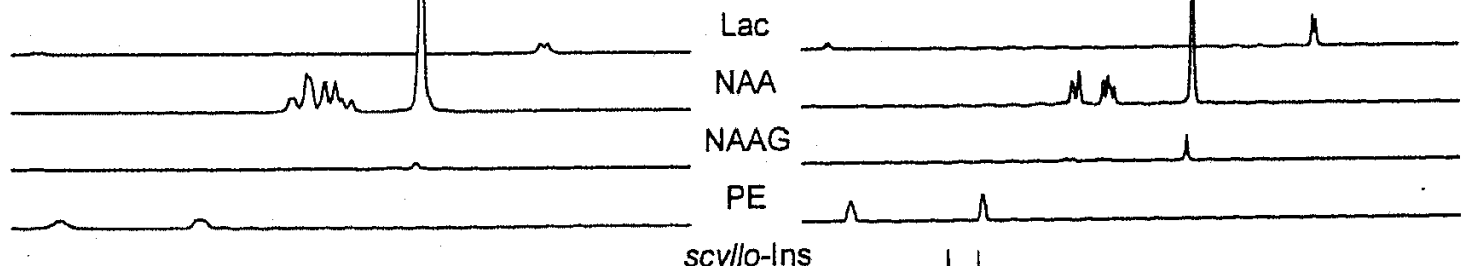
NAAG

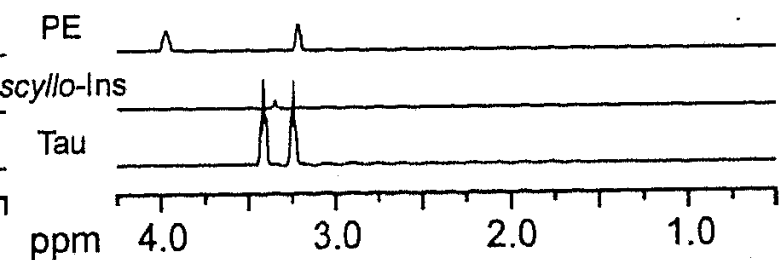

Figure 6. LCModel analysis of in-vivo 'H NMR spectra at 4 Tesla (human brain) and $9.4 \mathrm{~T}$ (rat brain). Shown is the in-vivo spectrum (top), the LCModel fit and fit residual, along with the estimated baseline and all contributions from the basis set to the in-vivo spectrum. 


\subsection{Glucose Concentration, and Distribution in the Brain: Transport Physiology}

Glucose is the single most important substrate required for normal brain function, and the brain relies on a continuous import of glucose from the blood, which must occur across the blood-brain barrier. Glucose transport rates into the brain are thus indicative of the maximal sustainable rate of glucose consumption, $\mathrm{CMR}_{\mathrm{glc}}$. Aside from lactate and glycogen (12), brain glucose is the only sizable kinetic pool upstream of acetyl-CoA, and the size of the brain glucose pool and its physical distribution space is important for derivation of absolute metabolic fluxes and quantitative positron emission tomography studies [9,77]. It has been shown that steady-state glucose transport kinetics can be derived from the relationship between brain and plasma glucose $[9,34,77,78]$.

Glucose concentrations have been measured using ${ }^{1} \mathrm{H}$ and ${ }^{13} \mathrm{C}$ NMR spectroscopy $[34,79-84]$. For a $\mathrm{CH}$ group with homonuclear $J$-coupling, the sensitivity advantage of ${ }^{1} \mathrm{H}$ compared to ${ }^{13} \mathrm{C}$ is expected to be only $2-3$ fold. The proximity of the ${ }^{1} \mathrm{H}$ resonances of glucose to that of water makes the measurement of brain glucose using ${ }^{1} \mathrm{H}$ NMR spectroscopy difficult, especially for ${ }^{1} \mathrm{H}$ resonance [60]. Therefore, in cases where metabolism is followed by ${ }^{13} \mathrm{C}$ NMR, it may be advantageous to measure brain glucose content as well.

When measuring brain glucose transport kinetics, the magnitude of the physical distribution volume, i.e., the volume in the brain into which glucose can diffuse, can affect the interpretation of the derived glucose transport kinetics [85-87]. The physical distribution volume of glucose has been assumed to equal the brain's water phase based on radiotracer and slice diffusion experiments, suggesting that transport of glucose across cell membranes is diffusion-limited [87], although some fraction of the aqueous space might be more accessible on a short time scale [88]. Interestingly, a study concluded that the physical distribution volume of glucose must be large and consistent with that of the brain's water phase from the delayed change in brain glucose following a step function in plasma glucose [89]. In a recent study the diffusion behavior of glucose was measured using diffusionweighted ${ }^{1} \mathrm{H}$ NMR spectroscopy [84], and it was found that glucose had a distinctly detectable signal even at very high diffusion weighting (Figure 7), consistent with a predominantly restricted diffusion behavior, reflecting the primarily intracellular origin of the glucose signal. However, the diffusion signal characteristic was different from compounds whose concentration is almost exclusively intracellular, but similar to that of lactate, whose extracellular concentration under normal circumstances is probably similar to whole brain lactate concentrations. From the diffusion behavior of the glucose signal it was concluded that approximately $20 \%$ of the NMR signal of glucose originated from the extracellular compartment, which reinforced the notion of a high physical distribution volume for glucose [90].

Traditionally, glucose transport kinetics have been analyzed with a model of brain glucose transport that was based on standard Michaelis-Menten kinetics. However, Michaelis-Menten kinetics is based on the assumption that the initial 


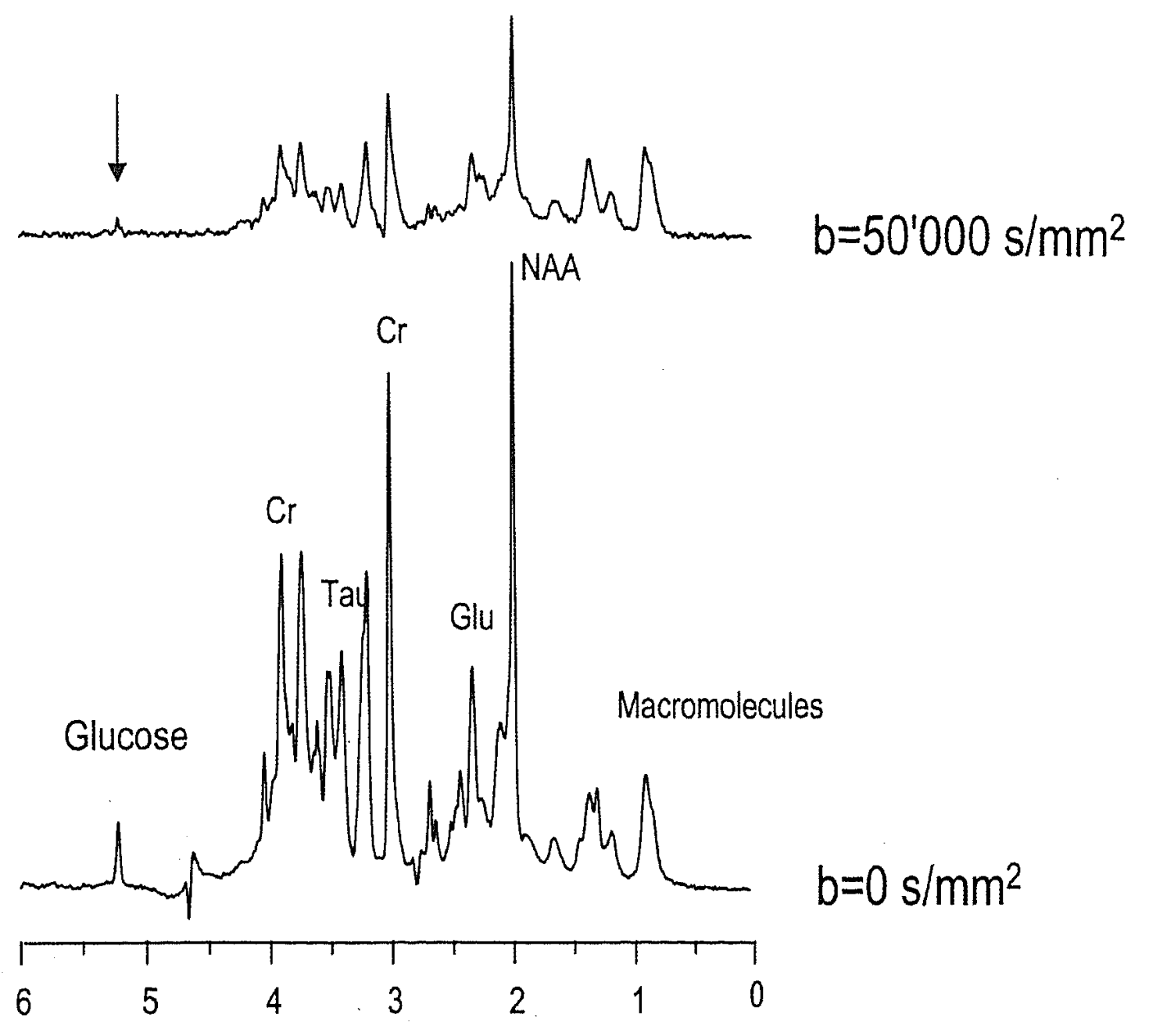

Figure 7. Diffusion-weighted signal of glucose and other metabolites [84]. Signals are strongly reduced at the high $b$ value (diffusion time was $120 \mathrm{~ms}$ ), more so than the macromolecule signal (at, e.g., $0.9 \mathrm{ppm}$ ). The detection of the glucose signal at $5.23 \mathrm{ppm}$ (arrow) indicates that a substantial fraction of the glucose is in intracellular space.

rate of unidirectional product formation is measured, e.g., immediately after substrate and enzyme have been combined. This experimental condition would require elimination of the brain glucose, which is difficult to achieve without interfering with normal brain function. Hence it is reasonable to expect that reversible Michaelis-Menten kinetics is a more appropriate formalism in describing brain glucose transport. Such a model has been proposed [34,91]. It was shown that one implication of the reversible model of brain glucose transport is that the relationship between brain and plasma glucose is linear [34]. Many measurements of brain glucose content as a function of plasma glucose have in the meantime corroborated the observation that brain glucose concentrations are a linear function of plasma glucose [78,81-83], and such a case is illustrated in Figure 8 for two different anesthetic regimes $-\alpha$-chloralose and pentobarbital. These studies indicated that decreased electrical activity and thus decreased energy metabolism resulted in in- 
creased brain glucose concentrations. The increment in brain glucose was consistent with an approximately twofold reduction in brain glucose utilization under pentobarbital anesthesia relative to $\alpha$-chloralose anesthesia. The presence of a sizable concentration gradient between brain and plasma glucose implies that net glucose uptake (i.e., glucose consumption at steady state) was appreciable even under conditions close to isoelectricity.

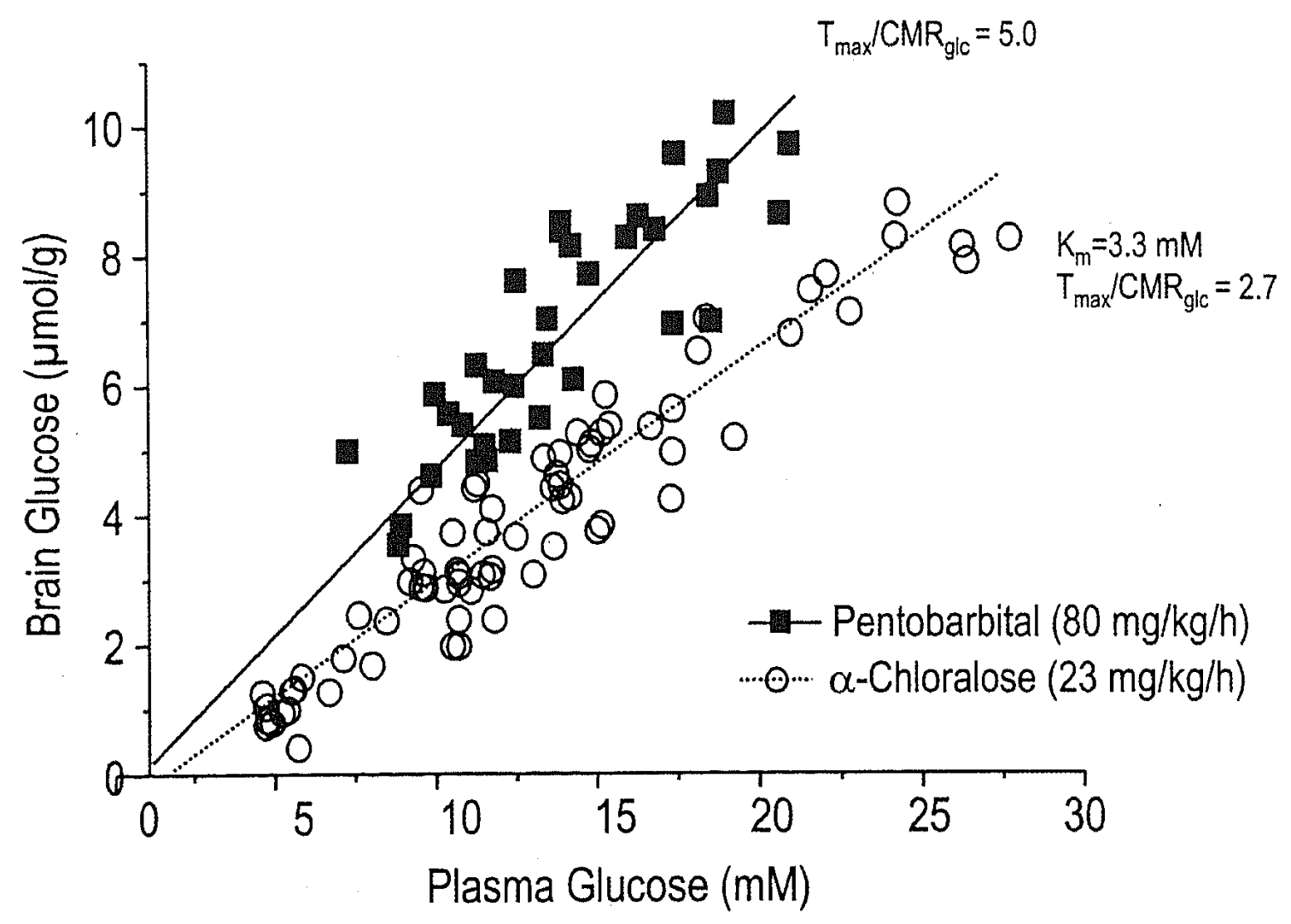

Figure 8. Brain glucose transport kinetics from the measurement of the brain glucose content as a function of plasma glucose concentration. (A) Demonstration of a linear relationship between brain and plasma glucose concentrations, as well as the effect of increased anesthesia (decreased electrical activity) on brain glucose content in vivo. Reprinted with permission from IY Choi, H Lei, R Gruetter. 2002. Effect of deep pentobarbital anesthesia on neurotransmitter metabolism in vivo: on the correlation of total glucose consumption with glutamatergic action. J Cereb Blood Flow Met 22:1343-1351. Copyright (C) 2002, International Society for Cerebral Blood Flow \& Metabolism.

The importance of measuring the brain glucose concentration can be appreciated from its role in regulating brain glucose metabolism: Glucose becomes ratelimiting for metabolism when its concentration approaches that of the $K_{\mathrm{m}}$ of the first step in its metabolism, which is in the brain phosphorylation mostly by hexokinase. Since the $K_{\mathrm{m}}$ of brain hexokinase is very low $(\sim 50 \mu \mathrm{M})$ and NMR sensitivity in vivo generally is too low to detect such small concentrations of glucose, brain glucose concentrations measured by NMR that are close to zero indicate metabolism that is limited by the glucose available to the brain cell. The general con- 
sensus is that brain glucose transport is not rate-limiting for metabolism under normal circumstances. We have recently shown in the conscious human and the $\alpha$ chloralose anesthetized rat that the maximal sustainable rate of glucose consumption is approximately $60-90 \%$ above the basal rate of glucose metabolism and brain glucose is therefore not rate-limiting $[34,78]$. This may, however, not be the case under conditions of extreme metabolic activation or during hypoglycemia [92].

Previously, models of brain glucose transport have been evaluated at normal or hyperglycemic conditions only [34,77,81-83]. A recent study extended the brain glucose concentration measurements to hypoglycemia using ${ }^{13} \mathrm{C}$ NMR spectroscopy [78]. The concentrations measured by ${ }^{13} \mathrm{C}$ NMR were found to be in excellent agreement to those predicted by the reversible Michaelis-Menten model as well as those measured by ${ }^{1} \mathrm{H}$ NMR spectroscopy [78]. Interestingly, when the brain glucose concentration approached zero, CBF was acutely increased [78] and glycogen degradation started [93], all of which points to brain glucose being important in activating cerebral defenses against a deficiency in fuel supply.

\subsection{Brain Glycogen, an Endogenous Energy Store}

Additional fuel can be provided to brain cells during, e.g., hypoglycemia, from glycogen, which is present in the brain in measurable quantities and appears to be essential for brain function. Brain glycogen is typically present in quantities that exceed those of tissue glucose in the brain. Similar to glucose, brain glycogen is rapidly eliminated in postmortem tissue $[12,94,95]$; therefore, its direct biochemical measurement is difficult $[96,97]$. Localized ${ }^{13} \mathrm{C}$ NMR spectroscopy has the unique capability of following brain glycogen metabolism longitudinally, employing a much smaller number of animals than would be used with biochemical extraction. The rate of brain glycogen degradation during hypoglycemia accounted for the majority of the glucose supply deficit during the hypoglycemic period [93]. Together with the apparent stability of glycogen in the non-stimulated brain at euor hyperglycemia $[12,98]$, these data suggest that brain glucose plays an important regulatory role in cerebral glycogenolysis. These studies also showed that brain glycogen increased above the basal level and beyond following a single episode of hypoglycemia [93]. This rebound or super-compensation of brain glycogen may result in increased neuroprotection. It has been proposed that brain glycogen metabolism may be a factor involved in the mechanism of the hypoglycemia unawareness syndrome observed clinically in patients with type I diabetes [93,98], perhaps through the enhanced neuroprotective effect of increased brain glycogen.

Thus glycogen likely is a viable and important store of glucose equivalents in the brain, whose metabolism is affected by hormones, neurotransmitters and second messengers [99].

One obvious investigational power of NMR spectroscopy is its applicability to animal models and humans alike. Because all previous studies measured brain glycogen metabolism in animals, the question remained as to whether brain glycogen 
$\left[1-{ }^{13} \mathrm{C}\right] \mathrm{D}$-Glucose
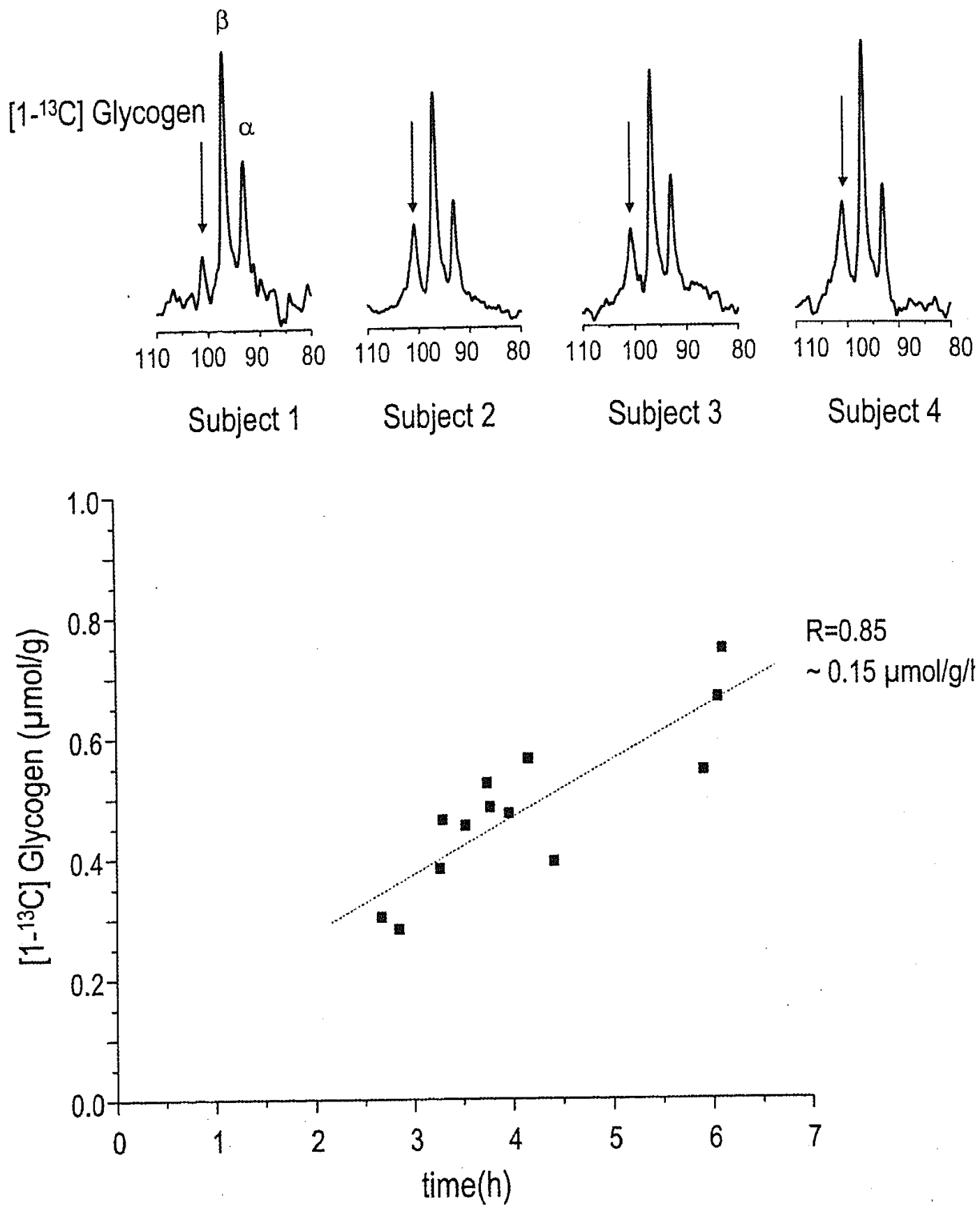

Figure 9. Measurement of glycogen in the human brain. (A) Demonstrates detection of the brain glycogen signal in four different subjects (arrows) along with the glucose $\mathrm{C} 1$ resonances. Shown is the spectral region containing the glycogen $\mathrm{C} 1$ and glucose $\mathrm{C} 1$ resonances. (B) The increase in the quantified glycogen $\mathrm{C} 1$ signal represents the accumulation of $\left[1-{ }^{13} \mathrm{C}\right]$ glycogen, which occurred at an extremely slow rate on the order of $0.15 \mu \mathrm{mol} / \mathrm{g} / \mathrm{h}$ in the human brain, as illustrated in the graph containing measurements from three different studies. Reprinted with permission from G Oz, PG Henry, ER Seaquist, R Gruetter. 2003. Direct, noninvasive measurement of brain glycogen metabolism in humans. Neurochem Int 43:323329. Copyright () 2003, Elsevier. 
metabolism may be faster in the conscious human brain. Brain glycogen metabolism had never been measured in the human brain and ${ }^{13} \mathrm{C}$ NMR is the only technique that can provide this insight. We have recently adapted the localization method (see above on localization methods) for measuring brain glycogen in humans and demonstrated that a reproducible measurement of the brain glycogen signal was indeed possible in the human brain [14], illustrated in Figure 9A. These initial results furthermore demonstrated that brain glycogen metabolism was extremely slow in subjects measured in the awake, resting condition (Figure 9B). This observation was in excellent agreement with previous studies showing that under the conditions of this study (plasma glucose at euglycemia or higher with concomitant hyperinsulinemia) the brain glucose concentration is well above the $K_{\mathrm{m}}$ of hexokinase $[9,34,83]$, thereby eliminating the need for appreciable glycogen activation. In fact, the flux through glycogen synthase was estimated at 0.1-0.2 $\mu \mathrm{mol} / \mathrm{g} / \mathrm{h}$. As a consequence, a brain glycogen pool of a few $\mathrm{mM}$ is expected to have a turnover time on the order of several days to a week. These findings suggest that glycogen metabolism is a negligible factor in the energy metabolism of the conscious unstimulated human brain at euglycemia and above.

\subsection{Compartmentalized Oxygen Metabolism in Brain}

The question of whether there is tight coupling between glucose and oxygen consumption in the brain has become of paramount importance. The landmark study by Fox and Raichle in the late 1980s suggested that there is indeed a large increase in glucose metabolism that exceeds the changes in oxygen metabolism [100]. The concept of uncoupled oxygen metabolism has been supported by studies reporting small increases in brain lactate during focal activation [101,102] that are very difficult to perform [103]. The relatively small magnitude of change in brain lactate $[101,102,104]$ is difficult to reconcile with the reported large uncoupling between oxygen and glucose consumption [105], and explanations linking the lactate increase to brain glycogen [106] at present appear unlikely (see above). To address this question, it is useful to measure the TCA cycle activity in the brain. In intact tissue the transfer of ${ }^{13} \mathrm{C}$ label into the glutamate pool has been linked to TCA cycle flux. The rate of label incorporation indicated a significant difference in the rate of glutamate labeling with activation, but oxygen consumption increased at most by $30 \%$, which is approximately half of the cerebral blood flow increase measured using this stimulation paradigm [107]. This study supported the concept that oxygen consumption increases are less than the associated cerebral blood flow increases, leading to a net decrease in deoxyhemoglobin content during focal activation, which forms the basis of blood-oxygen-level-dependent functional MRI [108].

Measurements of Krebs cycle flux from the flow of label from glucose $\mathrm{C} 1$ to glutamate $\mathrm{C} 4$ are affected by transport across the highly charged inner mitochondrial membrane. It is likely that such transport is controlled, since rapid transport of acids is unlikely and would interfere with chemiosmosis [109]. Indeed, this ex- 
change rate, $V_{\mathrm{x}}$, was found to be comparable to the flux through pyruvate dehydrogenase, $V_{\mathrm{PDH}}$, in most recent studies of the intact brain $[48,81,110]$, consistent with what has been reported in most other tissues [76,111-113]. Many, but not all, investigators have found that $V_{\mathrm{x}}$ is on the order of $V_{\mathrm{PDH}}$, which implied that the malate-aspartate shuttle may be a major mechanism mediating the exchange of label across the mitochondrial membrane $[30,48,81,110,111,114-117]$.

\subsubsection{Glutamatergic Neurotransmission and Glial Energy Metabolism}

Brain metabolism is exquisitely compartmentalized, with at least two major compartments (attributed to the "neuronal" and "glial" compartment) that are differentiated by the size of the respective glutamate pools associated with the Krebs cycle and that are metabolically linked by the so-called glutamate-glutamine cycle $[118,119]$. The scheme in Figure 10 summarizes some of the salient features that have been exploited at high field in modeling brain metabolism.

Key to cerebral compartmentation is inactivation of neurotransmitter glutamate by uptake into perisynaptic astrocytes and conversion into electrophysiologically inactive glutamine, which then diffuses back to the neuron [120-123]. Clearly, this mechanism implies a much more active role for astrocytes than is conventionally assumed, since the conversion of neurotransmitter glutamate to glutamine invokes glial energy metabolism [124-126]. The neuron-astrocyte pair thus has to be considered the functional unit (the "tripartite synapse" containing the pre- and postsynaptic neuron and the astrocyte) intimately involved in achieving chemical transmission, as has been proposed in the last decade by Magistretti and others [127-129].

The link between astrocytes and neurons is generally accepted from metabolic and neurophysiological standpoints [130], yet differences exist as to the precise relationship between the metabolic rates and the specific energetics involved $[48,81,117,131,132]$. The bulk of investigations suggest that non-signaling energy metabolism is approximately a third to half that at resting (non-stimulated) conditions $[117,133]$. Nonetheless, the controversial hypothesis put forth by Shulman et al. $[131,134]$ linking glucose metabolism with glutamatergic action is intriguing as it emphasizes further the coupling between neurons and glia at the level of energy metabolism as put forward earlier by others (see, e.g., [129]).

Astrocytes have significant oxidative capacity to generate ATP, which can be substantiated with two experimental observations. First, anaplerosis, a mitochondrial reaction confined to the glial compartment, mediated by pyruvate carboxylase, is now generally accepted to have significant activity in vivo $[11,13,48,116,117$, $135,136]$. To the extent that anaplerosis leads to net glutamine synthesis (via pyruvate carboxylase) in the glial compartment, a significant net synthesis of ATP occurs oxidatively, thereby generating approximately a third of the ATP per glucose molecule compared to that from complete glucose oxidation [116]. Second, the presence of significant oxidative metabolism in astrocytes stems from the fact that acetate is known to be exclusively metabolized in astrocytes by the TCA cycle $[137,138]$, which can only occur by oxidative metabolism [139]. 


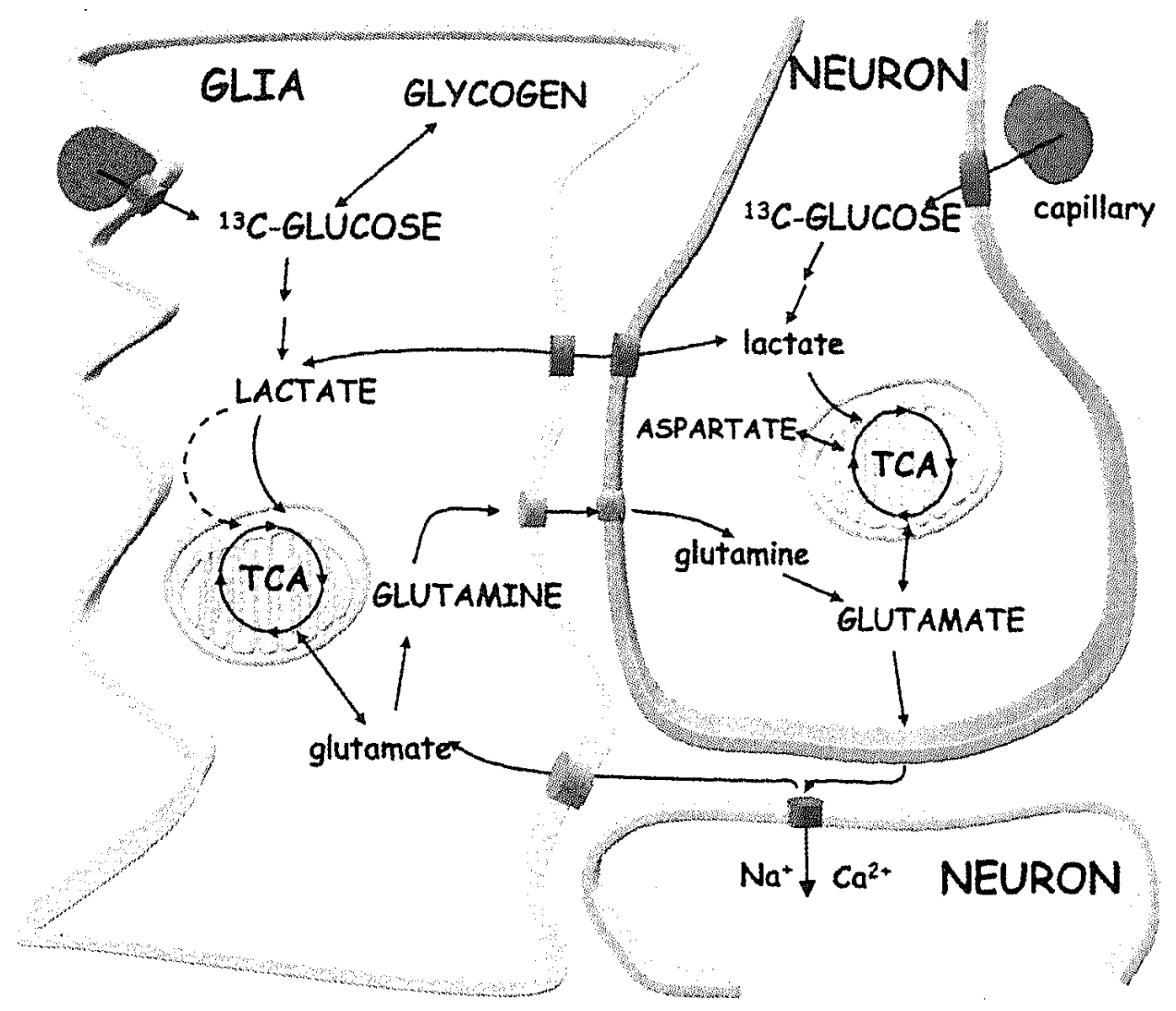

Figure 10. Compartmentation of cerebral neurotransmitter and amino acid metabolism studied via labeling from glucose at, e.g., the $\mathrm{Cl}$ position. The glial compartment is on the left and the neuronal on the right. Anaplerosis, mediated by pyruvate carboxylase (dashed arrow), glutamine synthetase, as well as glycogen and its metabolism, are localized to the glial compartment in the adult brain. Most of the glutamine is in the glial compartment and most of the glutamate and aspartate in the neuronal compartment. The metabolites in caps give rise to NMR signals we have measured in vivo. See attached CD for color version.

\subsection{Studies of Individual Subjects}

The improvements in in-vivo NMR spectroscopy at high fields (highlighted in the first half of the chapter) all translate into increased statistical power, mainly due to sensitivity improvements. Thus smaller volumes can be studies with linear dimensions decreased by the third root of the sensitivity gains, or measurement times can be shortened by the square of the sensitivity improvements.

\subsubsection{Separate Quantification of Glutamate and Glutamine: Hepatic Encephalopathy}

As stated earlier in the chapter, one obvious benefit of higher fields is the clearly increased spectral resolution of especially $J$-coupled resonances, such as glutamate and glutamine. While commonly small changes in brain glutamine are difficult to discern at low fields, such changes in brain glutamine are visually ap- 
parent in individual subjects at 4 Tesla [3]. In a study of pediatric patients at 4 Tesla with subclinical hepatic encephalopathy, the increase in brain glutamine was easily detectable (Figure 11). Furthermore, the tight reproducibility of the ${ }^{1} \mathrm{H}$ NMR spectra allowed to conclude that the increased error in the patient group (Figure 11) was not due to increased quantification errors but rather reflected differences between individual patients. It is of interest to note that while brain glutamine showed distinct and up to a several-fold increase in subclinical HE, the combined Glu and Gln signal (Glx) exhibited only minor variations, as the Gln changes were masked by the much more concentrated Glu signal.

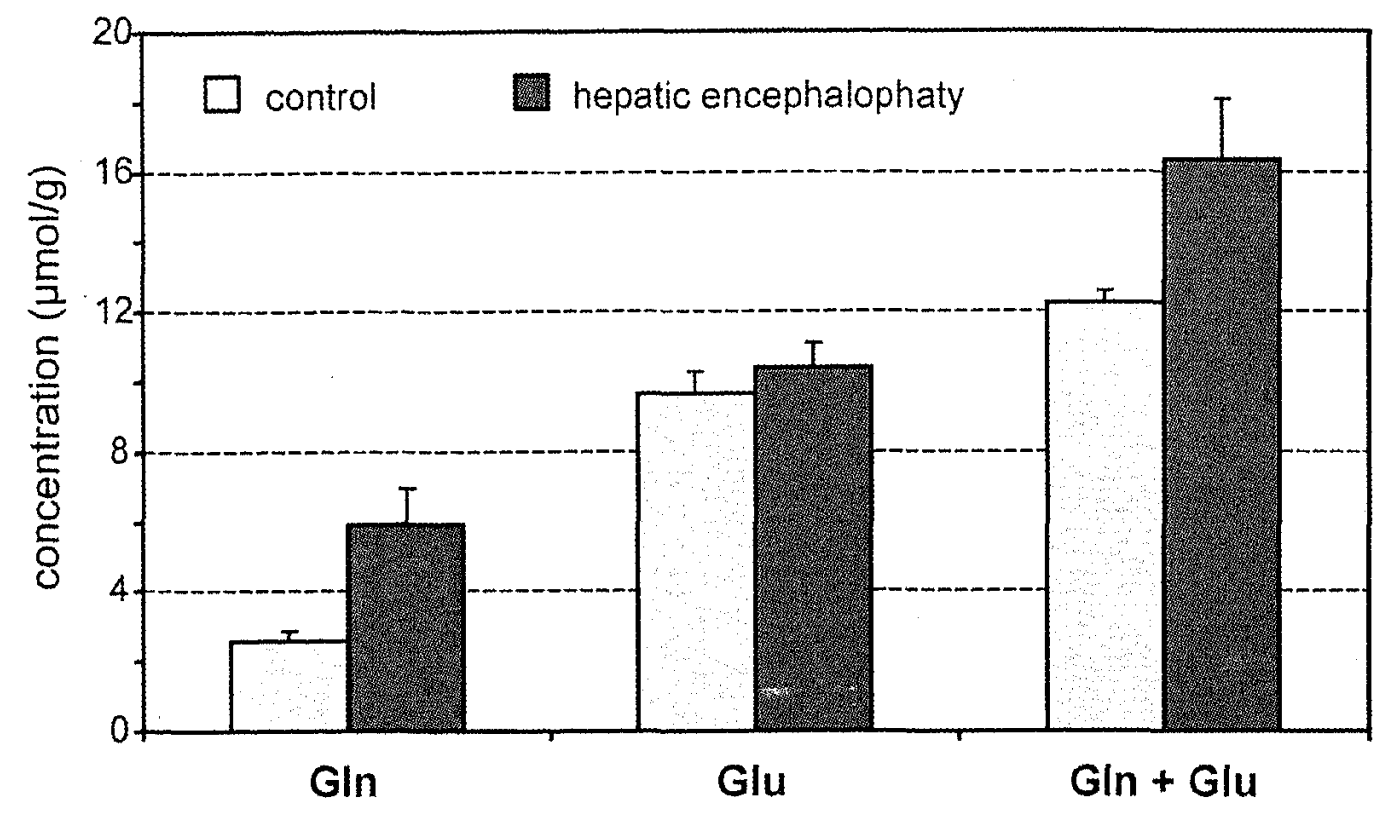

Figure 11. Changes in Glu, Gln, and Glx (Glu + Gln) with HE (bar graph), illustrating the increased statistical power when separating the change in Gln from that of Glu.

\subsubsection{Disease Monitoring in Pediatric Patients: Adrenoleukodystrophy}

The increases in sensitivity with higher field predispose the method to increased use in especially pediatrics, where subject compliance is difficult to achieve, and hence shorter acquisition times and potential for correction of motion effects can become of paramount importance. Using single-shot acquisition, it was shown that robust and reliable quantification of 12 metabolites was possible in unsedated patients with ALD [59]. Furthermore, in relatively small volumes in white matter (WM), profound changes were observed in the neurochemical profile of lesions (Figure 12). Of special interest was the clear and unambiguous separation of 'H NMR spectra obtained from lesioned WM, which were unambiguously separated from normal appearing WM, with several-fold differences in some compounds. These observations point to the ability of ${ }^{1} \mathrm{H}$ NMR spectroscopy to characterize disease onset in prospective lesion sites, long before structural damage is 
evident in MRI. In several cases, changes in the neurochemical profile indeed preceded the appearance of hyperintense lesions in $T_{2}$-weighted MRI.

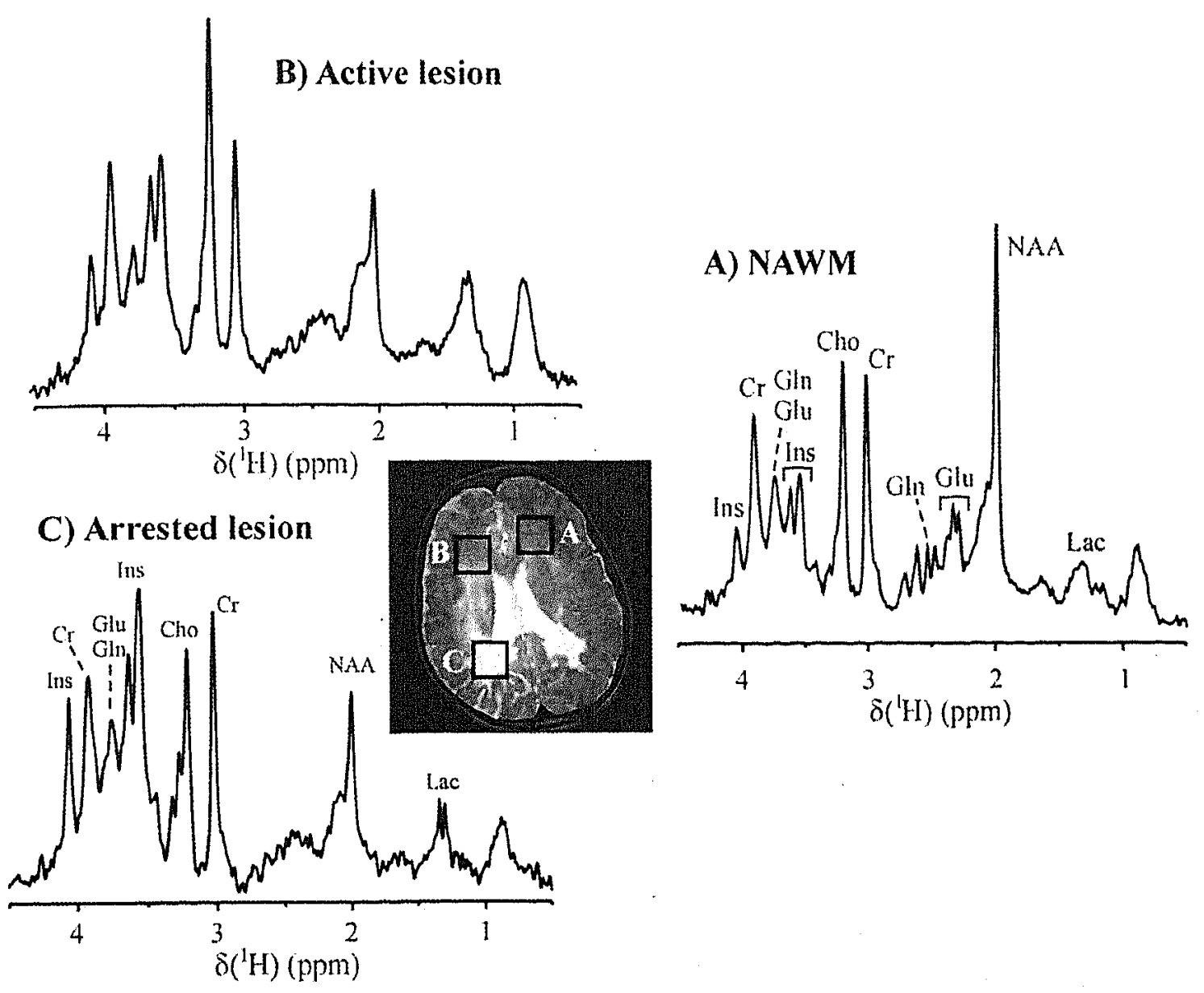

Figure 12. In-vivo NMR spectroscopy at high field can be used for therapy monitoring in individual patients. $T_{2}$-weighted RARE image (TE $=60 \mathrm{~ms}$, echo train length of 8 ) at 4 Tesla depicting the status of lesions in a patient with the cerebral form of ALD following treatment. Three distinct states of the diseases are discernible in the neurochemical profile: (A) normalappearing white matter, (B) an active lesion that emerged after treatment, and (C) an arrested lesion.

\subsubsection{Detection of Two Important Antioxidants: Glutathione and Vitamin C}

Two important antioxidant compounds - glutathione and Vitamin C (ascorbate) - are present in the human brain in sufficient amounts to be detectable in vivo by NMR spectroscopy. The ability to measure glutathione was demonstrated using short-echo spectroscopy at 9.4 Tesla in the rat brain [5]. Changes in glutathione concentration following administration of quinolinic acid in a chemical model of Huntington disease, measured by NMR spectroscopy [26], were in excellent agreement with biochemical studies in the same animal model [140]. The measurement of GSH using short-echo spectroscopy in combination with LCModel analysis is thus likely a robust measurement; however, even at 9.4 Tesla GSH lacks a clearly resolved peak. As pointed out above, spectral editing stands to gain from 
the increased spectral specificity of frequency-selective editing pulses at high fields. The ability of spectral editing to measure brain glutathione concentrations was supported by initial results obtained at 1.5 Tesla using multiple-quantum editing [61]. However, since the homonuclear $J$-coupling is largely independent of field strength, the contribution of the co-edited NAA aspartyl resonance must be taken into account, thereby complicating the analysis of GSH-edited spectra at low fields. In contrast, it was recently shown that using difference editing, it is possible at 4 Tesla to detect a resolved signal from GSH in human brain [32] (Figure 5). Because of its complex spin system, the signal of GSH exhibited a complex spectral pattern that was nevertheless possible to quantify using LCModel analysis, yielding a concentration that is in excellent agreement with that obtained by short-echo spectroscopy [32].

The spin system of ascorbate (Vitamin C), on the other hand, is spread over a narrow chemical shift range and more difficult to measure, due to its close proximity to the water resonance. However, the detection of ascorbate is aided at high fields by two factors. First, the increased spectral resolution at higher field does result in improved water suppression and thus a more reliable detection of resonances close to water. Second, the coupled spin system of ascorbate is restricted to a narrow spectral range of $0.8 \mathrm{ppm}$, making the selective editing by any standard difficult. Nonetheless, with judicious choice of experimental parameters it was shown that the reproducible and reliable detection and quantification of ascorbate was possible at 4 Tesla in the human brain (Figure 13), yielding concentrations on the order of $1.3 \mathrm{mM}$, in excellent agreement with postmortem analysis [141]. Ascorbate has been mainly ascribed to the neuronal compartment, whereas glutathione appears to have a mainly astrocytic localization, suggesting that the two antioxidant systems act in tandem to protect the brain from oxidative damage. The ability to measure both systems using high field NMR spectroscopy opens the possibility to comprehensively measure cerebral responses to oxidative stress.

\section{OUTLOOK}

The increased sensitivity at high field is likely to allow the measurement of many neurochemicals in small volumes in individual patients, leading to the potential to follow disease progression and therapy. It is important to recognize in this context that high field alone, without concomitant technical improvements and care, may not allow one to garner these advantages, implying that substantial improvements aside from brute increments in $B_{0}$ are likely to enhance the biomedical research power of this investigative tool.

The review presented in this chapter illustrates that with appropriate technical improvements NMR spectroscopy at high-fields can and already has made contributions to our understanding of brain metabolism and function, e.g., understanding glucose transport, measuring compartmentalized brain glutamate and glutamine metabolism, brain glycogen metabolism, and the measurement of important antioxidants. In addition, the ability to quantify brain glycogen metabolism has led to the postulation that brain glycogen is an important store of glucose equivalents. 


\section{Human In Vivo Detection at 4T}

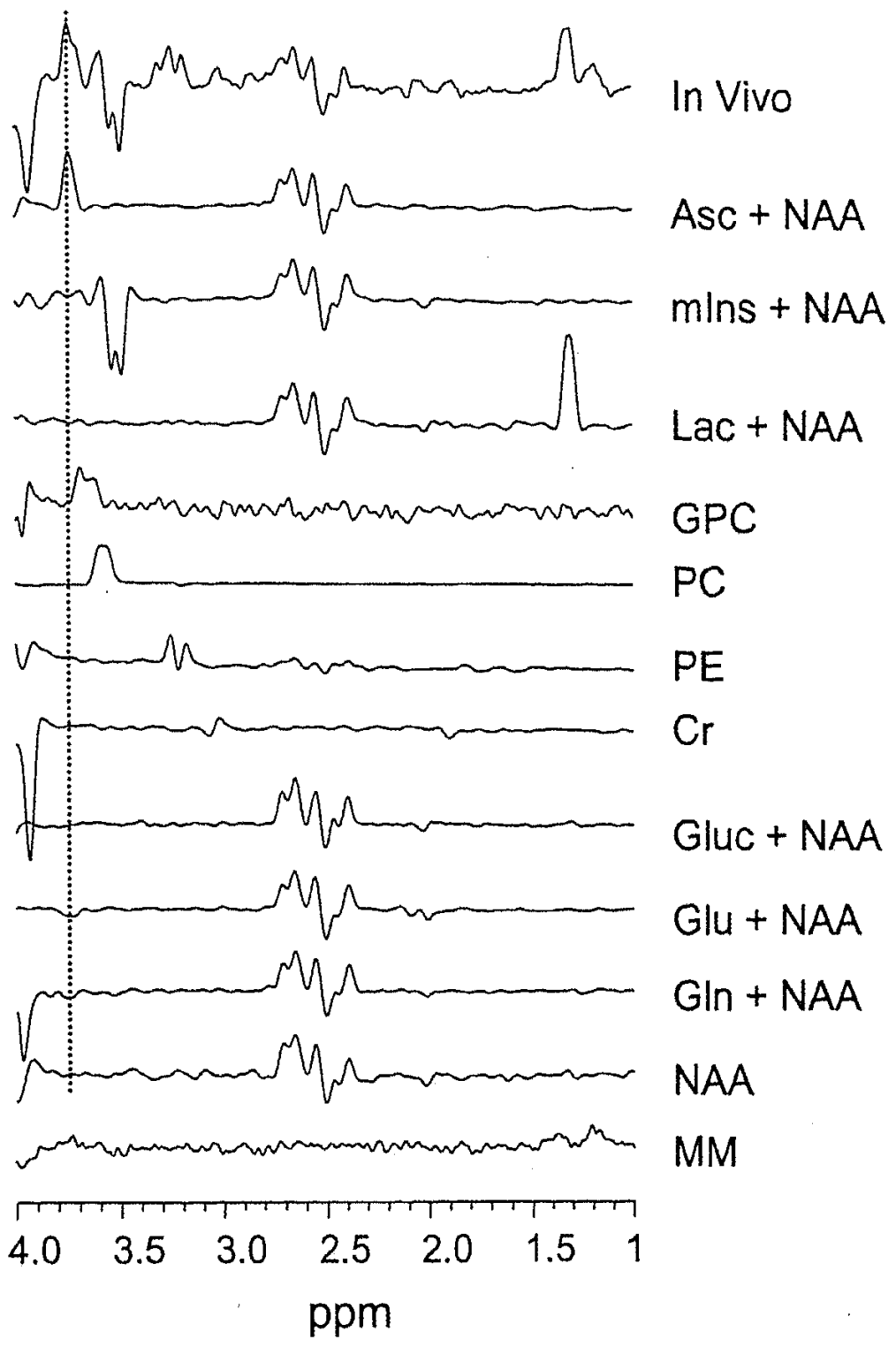

Figure 13. Assignment of in-vivo 'H NMR signal of Vitamin $\mathrm{C}$ in human brain. Reprinted with permission from Terpstra M, Gruetter R. 2004. 'H NMR detection of vitamin C in human brain in vivo. Magn Reson Med 51:225-229. Copyright (C 2004, John Wiley \& Sons Inc.

\section{ACKNOWLEDGMENTS}

The encouragement and support from colleagues at the Center for MR Research is appreciated. Partly supported by the Centre d'Imagerie BioMedicale (CIBM), research partnership in the Lausanne-Geneva region, Switzerland. Supported in part by grants from the U.S. Public Health Service, NIH R21DK58004 (RG), R01NS38672 (RG), R01NS42005 (RG), R21NS45119 (RG), P41RR08079, 
M01RR00400, and the Whitaker (RG), Juvenile Diabetes Research (RG), and Keck Foundations.

\section{REFERENCES}

1. Hoult D, Richards R. 1976. The signal-to-noise ratio of the nuclear magnetic resonance experiment. J Magn Reson 24:71-85.

2. Barker PB, Hearshen DO, Boska MD. 2001. Single-voxel proton MRS of the human brain at 1.5T and 3.0T. Magn Reson Med 45:765-769.

3. Gruetter R, Weisdorf SA, Rajanayagan V, Terpstra M, Merkle H, Truwit CL, Garwood $\mathrm{M}$, Nyberg SL, Ugurbil K. 1998. Resolution improvements in in vivo ${ }^{1} \mathrm{H}$ NMR spectra with increased magnetic field strength. J Magn Reson 135:260-264.

4. Tkac 1, Rao R, Georgieff MK, Gruetter R. 2003. Developmental and regional changes in the neurochemical profile of the rat brain determined by in vivo $1 \mathrm{H}$ NMR spectroscopy. Magn Reson Med 50:24-32.

5. Pfeuffer J, Tkac I, Provencher SW, Gruetter R. 1999. Toward an in vivo neurochemical profile: quantification of 18 metabolites in short-echo-time ${ }^{1} \mathrm{H}$ NMR spectra of the rat brain. J Magn Reson 141:104-120.

6. Tkác I, Starcuk Z, Choi I-Y, Gruetter R. 1998. Ultra-short Echo-time "Dehydrated" in vivo ${ }^{1} \mathrm{H}$ NMR Spectroscopy. Proc Int Soc Magn Reson Med 1760.

7. Van Cauteren M, Miot F, Segebarth CM, Eisendrath H, Osteaux M, Willem R. 1992. Excitation characteristics of adiabatic half-passage RF pulses used in surface coil MR spectroscopy: application to ${ }^{13} \mathrm{C}$ detection of glycogen in the rat liver. Phys Med Biol 37:1055-1064.

8. Gruetter R, Rothman DL, Novotny EJ, Shulman RG. 1992. Localized ${ }^{13} \mathrm{C}$ NMR spectroscopy of myo-inositol in the human brain in vivo. Magn Reson Med 25:204-210.

9. Gruetter R, Novotny EJ, Boulware SD, Rothman DL, Mason GF, Shulman GI, Shulman RG, Tamborlane WV. 1992. Direct measurement of brain glucose concentrations in humans by ${ }^{13} \mathrm{C}$ NMR spectroscopy. Proc Natl Acad Sci USA 89:1109-1112.

10. deGraaf R, Luo Y, Terpstra M, Garwood M. 1995. Spectral editing with adiabatic pulses. J Magn Reson B 109:184-193.

11. Gruetter R, Seaquist E, Kim S-W, Ugurbil K. 1998. Localized in vivo ${ }^{13} \mathrm{C}$ NMR of glutamate metabolism: initial results at 4 Tesla. Dev Neurosci 20:380-388.

12. Choi IY, Tkac I, Ugurbil K, Gruetter R. 1999. Noninvasive measurements of [1${ }^{13} \mathrm{C}$ ]glycogen concentrations and metabolism in rat brain in vivo. $J$ Neurochem 73:1300-1308.

13. Shen J, Petersen KF, Behar KL, Brown P, Nixon TW, Mason GF, Petroff OA, Shulman GI, Shulman RG, Rothman DL. 1999. Determination of the rate of the glutamate/glutamine cycle in the human brain by in vivo ${ }^{13} \mathrm{C}$ NMR. Proc Natl Acad Sci USA 96:8235-8240.

14. Oz G, Henry PG, Seaquist ER, Gruetter R. 2003. Direct, noninvasive measurement of brain glycogen metabolism in humans. Neurochem Int 43:323-329.

15. Henry PG, Tkac I, Gruetter R. 2003. ${ }^{1} \mathrm{H}$-localized broadband 13C NMR spectroscopy of the rat brain in vivo at 9.4 T. Magn Reson Med 50:684-692.

16. Collins CM, Liu W, Wang J, Gruetter R, Vaughan JT, Ugurbil K, Smith MB. 2004. Temperature and SAR calculations for a human head within volume and surface coils at 64 and $300 \mathrm{MHz}$ J Magn Reson Imag 19:650-656. 
17. van den Bergh AJ, van den Boogert HJ, Heerschap A. 1998. Calibration of the $1 \mathrm{H}$ decoupling field strength and experimental evaluation of the specific RF absorption rate in ${ }^{1} \mathrm{H}$-decoupled human ${ }^{13} \mathrm{C}-\mathrm{MRS}$. Magn Reson Med 39:642-646.

18. Bax A. 1983. A simple method for the calibration of the decoupler radiofrequency field strength. J Magn Reson 52:76-80.

19. Tkac I, Henry PG, Andersen P, Keene CD, Low WC, Gruetter R. 2004. Highly resolved in vivo ${ }^{1} \mathrm{H}$ NMR spectroscopy of the mouse brain at 9.4 T. Magn Reson Med 52:478-484.

20. Shen J, Rycyna RE, Rothman DL. 1997. Improvements on an in vivo automatic shimming method (FASTERMAP). Magn Reson Med 38:834-839.

21. Manabe A. 1994. Multi-angle projection shim (MAPShim): in vivo shim adjustment up to 2 nd order in 0.2 second sequence time. Proc Soc Magn Reson 765.

22. Gruetter R. 1993. Automatic, localized in vivo adjustment of all first- and second-order shim coils. Magn Reson Med 29:804-811.

23. Gruetter R, Boesch C. 1992. Fast, non-iterative shimming on spatially localized signals: in vivo analysis of the magnetic field along axes. J Magn Reson 96:323-334.

24. Gruetter R, Tkac I. 2000. Field mapping without reference scan using asymmetric echo-planar techniques. Magn Reson Med 43:319-323.

25. Tkac I, Starcuk Z, Choi I-Y, Gruetter R. 1999. In vivo 'H NMR spectroscopy of rat brain at $1 \mathrm{~ms}$ echo time. Magn Reson Med 41:649-656.

26. Tkac I, Keene CD, Pfeuffer J, Low WC, Gruetter R. 2001. Metabolic changes in quinolinic acid-lesioned rat striatum detected non-invasively by in vivo ${ }^{1} \mathrm{H}$ NMR spectroscopy. J Neurosci Res 66:891-898.

27. Ross BD, Bluml S, Cowan R, Danielsen E, Farrow N, Gruetter R. 1997. In vivo magnetic resonance spectroscopy of human brain: the biophysical basis of dementia. Biophys Chem 68:161-172.

28. Bluml S, Hwang JH, Moreno A, Ross BD. 2000. Novel peak assignments of in vivo ${ }^{13} \mathrm{C}$ MRS in human brain at 1.5 T. J Magn Reson 143:292-298.

29. Choi IY, Gruetter R. 2004. Dynamic or inert metabolism? Turnover of N-acetyl aspartate and glutathione from $\mathrm{D}-\left[1-{ }^{13} \mathrm{C}\right]$ glucose in the rat brain in vivo. $J$ Neurochem 91:778-787.

30. Gruetter R, Adriany G, Choi IY, Henry PG, Lei H, Oz G. 2003. Localized in vivo ${ }^{13} \mathrm{C}$ NMR spectroscopy of the brain. NMR Biomed 16:313-338.

31. Gruetter R, Adriany G, Merkle H, Andersen PM. 1996. Broadband decoupled, 'H localized ${ }^{13} \mathrm{C}$ MRS of the human brain at 4 Tesla. Magn Reson Med 36:659-664.

32. Terpstra M, Henry PG, Gruetter R. 2003. Measurement of reduced glutathione (GSH) in human brain using LCModel analysis of difference-edited spectra. Magn Reson Med 50:19-23.

33. Seaquist ER, Gruetter R. 1998. Identification of a high concentration of scyllo-inositol in the brain of a healthy human subject using ${ }^{1} \mathrm{H}$ and ${ }^{13} \mathrm{C}$ NMR. Magn Reson Med 39:313-316.

34. Gruetter R, Ugurbil K, Seaquist ER. 1998. Steady-state cerebral glucose concentrations and transport in the human brain. $J$ Neurochem 70:397-408.

35. Bluml S. 1999. In vivo quantitation of cerebral metabolite concentrations using natural abundance ${ }^{13} \mathrm{C}$ MRS at 1.5 T. J Magn Reson 136:219-225.

36. Sonnewald U, Gribbestad IS, Westergaard N, Nilsen G, Unsgard G, Schousboe A, Petersen SB. 1994. Nuclear magnetic resonance spectroscopy: biochemical evaluation of brain function in vivo and in vitro. Neurotoxicology 15:579-590. 
37. Leibfritz D. 1996. An introduction to the potential of ${ }^{1} \mathrm{H}-,{ }^{31} \mathrm{P}$ - and ${ }^{13} \mathrm{C}-\mathrm{NMR}$ spectroscopy. Anticancer Res 16:1317-1324.

38. Shaka. AJ, Keeler J, Freeman R. 1983. Evaluation of a new broadband decouling sequence: WALTZ-16. J Magn Reson 53:313-340.

39. Pfeuffer J, Tkac I, Choi I-Y, Merkle H, Ugurbil K, Garwood M, Gruetter R. 1999. Localized in vivo 'H NMR detection of neurotransmitter labeling in rat brain during infusion of $\left[1-{ }^{13} \mathrm{C}\right]$ D-glucose. Magn Reson Med 41:1077-1083.

40. De Graaf RA, Mason GF, Patel AB, Rothman DL, Behar KL. 2004. Regional glucose metabolism and glutamatergic neurotransmission in rat brain in vivo. Proc Natl Acad Sci USA 101(34):12700-12705.

41. Muller S, Beckmann N. 1989. ${ }^{13} \mathrm{C}$ spectroscopic imaging. A simple approach to in vivo ${ }^{13} \mathrm{C}$ investigations. Magn Reson Med 12:400-406.

42. van den Bergh AJ, van den Boogert HJ, Heerschap A. 1998. Heteronuclear cross polarization for enhanced sensitivity of in vivo ${ }^{13} \mathrm{C}$ MR spectroscopy on a clinical $1.5 \mathrm{~T}$ MR system. J Magn Reson 135:93-98.

43. Brown TR, Kincaid BM, Ugurbil K. 1982. NMR chemical shift imaging in three dimensions. Proc Natl Acad Sci USA 79:3523-3526.

44. Haupt CI, Schuff N, Weiner MW, Maudsley AA. 1996. Removal of lipid artifacts in ${ }^{1} \mathrm{H}$ spectroscopic imaging by data extrapolation. Magn Reson Med 35:678-687.

45. Hu X, Patel M, Chen W, Ugurbil K. 1995. Reduction of truncation artifacts in CSI by extended sampling using variable TR. J Magn Reson A 106:292-296.

46. Doddrell DM, Pegg DT, Bendall MR. 1982. Distortionless Enhancment of NMR signals by polarization transfer. J Magn Reson 48:323-327.

47. Merkle H, Wei H, Garwood M, Ugurbil K. 1992. B insensitive heteronuclear adiabatic polarizaton transfer for signal enhancement. J Magn Reson 99:480-494.

48. Gruetter R, Seaquist ER, Ugurbil K. 2001. A mathematical model of compartmentalized neurotransmitter metabolism in the human brain. Am J Physiol 281:E100-112.

49. Bomsdorf H, Roschmann P, Wieland J. 1991. Sensitivity enhancement in whole-body natural abundance ${ }^{13} \mathrm{C}$ spectroscopy using ${ }^{13} \mathrm{C} /{ }^{1} \mathrm{H}$ double-resonance techniques at 4 tesla. Magn Reson Med 22:10-22.

50. Overloop K, Vanstapel F, Vanhecke P. $1996 .{ }^{13} \mathrm{C}-\mathrm{NMR}$ relaxation in glycogen. Magn Reson Med 36:45-51.

51. Zang LH, Laughlin MR, Rothman DL, Shulman RG. $1990 .{ }^{13} \mathrm{C}$ NMR relaxation times of hepatic glycogen in vitro and in vivo. Biochemistry 29:6815-6820.

52. Choi IY, Tkac I, Gruetter R. 2000. Single-shot, three-dimensional "non-echo" localization method for in vivo NMR spectroscopy. Magn Reson Med 44:387-394.

53. Pan JW, Hetherington HP. 1996. J-refocused coherence transfer spectroscopy to observe GABA at 4.1 T. Proc Intl Soc Magn Reson Med 1229.

54. Tkac I, Andersen P, Adriany G, Merkle H, Ugurbil K, Gruetter R. 2001. In vivo ${ }^{1} H$ NMR spectroscopy of the human brain at 7 T. Magn Reson Med 46:451-456.

55. Tkac I, Andersen P, Adriany G, Gruetter R, Ugurbil K. 1999. 4-ms echo-time ${ }^{1} \mathrm{H}$ NMR spectra of human brain measured in a 4 Tesla $/ 90 \mathrm{~cm}$ magnet using a body gradient coil. Proc Int Soc Magn Reson Med, Poster M1612.

56. Tannus A, Garwood M. 1996. Improved performance of frequency-swept pulses using offset-independent adiabaticity. J Magn Reson A 120:133-137.

57. Garwood M, DelaBarre L. 2001. The return of the frequency sweep: designing adiabatic pulses for contemporary NMR. J Magn Reson 153:155-177. 
58. Hetherington HH, Pan JW, Mason GF, Ponder S, Twieg DB, Deutsch G, Mountz J, Pohost GM. 1994. 2D 'H spectrosocopic imaging of the human brain at 4.IT. Magn Reson Med 32:530-534.

59. Oz G, Tkac I, Charnas LR, Choi IY, Bjoraker KJ, Shapiro EG, Gruetter R. 2005. Assessment of adrenoleukodystrophy lesions by high field MRS in non-sedated pediatric patients. Neurology 64:434-441.

60. Gruetter R, Garwood M, Ugurbil K, Seaquist ER. 1996. Observation of resolved glucose signals in ${ }^{1} \mathrm{H}$ NMR spectra of human brain at 4 Tesla. Magn Reson Med 36:1-6.

61. Trabesinger AH, Weber OM, Duc CO, Boesiger P. 1999. Detection of glutathione in the human brain in vivo by means of double quantum coherence filtering. Magn Reson Med 42:283-289.

62. Rothman DL, Petroff OA, Behar KL, Mattson RH. 1993. Localized 'H NMR measurements of gamma-aminobutyric acid in human brain in vivo. Proc Natl Acad Sci USA 90:5662-5666.

63. Jouvensal L, Carlier PG, Bloch G. 1996. Practical implementation of single-voxel double-quantum editing on a whole-body NMR spectrometer: localized monitoring of lactate in the human leg during and after exercise. Magn Reson Med 36:487-490.

64. Behar KL, Rothman DL, Spencer DD, Petroff OAC. 1994. Analysis of macromolecule resonances in ${ }^{1} \mathrm{H}$ MR spectra of human brain. Magn Reson Med 32:294-302.

65. Hanstock CC, Coupland NJ, Allen PS. 2002. GABA X2 multiplet measured pre- and post-administration of vigabatrin in human brain. Magn Reson Med 48:617-623.

66. Marjanska M, Henry PG, Bolan PJ, Vaughan B, Seaquist ER, Gruetter R, Ugurbil K, Garwood M. 2005. Uncovering hidden in vivo resonances using editing based on localized TOCSY. Magn Reson Med 53:783-789.

67. Terpstra M, Ugurbil K, Gruetter R. 2002. Direct in vivo measurement of human cerebral GABA concentration using MEGA-editing at 7 Tesla. Magn Reson Med 47:10091012.

68. Mescher M, Merkle H, Kirsch J, Garwood M, Gruetter R. 1998. Simultaneous water suppression and editing in vivo. NMR Biomed 11:266-272.

69. Slotboom J, Boesch C, Kreis R. 1998. Versatile frequency domain fitting using time domain models and prior knowledge. Magn Reson Med 39:899-911.

70. Provencher SW. 1993. Estimation of metabolite concentrations from localized in vivo proton NMR spectra. Magn Reson Med 30:672-679.

71. Naressi A, Couturier C, Devos JM, Janssen M, Mangeat C, de Beer R, GraveronDemilly D. 2001. Java-based graphical user interface for the MRUI quantitation package. MAGMA 12:141-152.

72. de Beer R, van den Boogaart A, van Ormondt D, Pijnappel WW, den Hollander JA, Marien AJ, Luyten PR. 1992. Application of time-domain fitting in the quantification of in vivo ' $\mathrm{H}$ spectroscopic imaging data sets. NMR Biomed 5:171-178.

73. Provencher SW. 2001. Automatic quantitation of localized in vivo ${ }^{1} \mathrm{H}$ spectra with LCModel. NMR Biomed 14:260-264.

74. Mader I, Seeger U, Karitzky J, Erb M, Schick F, Klose U. 2002. Proton magnetic resonance spectroscopy with metabolite nulling reveals regional differences of macromolecules in normal human brain. J Magn Reson Imag 16:538-546.

75. Opstad KS, Provencher SW, Bell BA, Griffiths JR, Howe FA. 2003. Detection of elevated glutathione in meningiomas by quantitative in vivo ${ }^{1} \mathrm{H}$ MRS. Magn Reson Med 49:632-637. 
76. Garcia-Martin ML, Garcia-Espinosa MA, Ballesteros P, Bruix M, Cerdan S. 2002. Hydrogen turnover and subcellular compartmentation of hepatic $\left[2-{ }^{13} \mathrm{C}\right]$ glutamate and $\left[3-{ }^{13} \mathrm{C}\right]$ aspartate as detected by ${ }^{13} \mathrm{C}$ NMR. J Biol Chem 277:7799-7807.

77. Mason GF, Behar KL, Rothman DL, Shulman RG. 1992. NMR determination of intracerebral glucose concentration and transport kinetics in rat brain. $J$ Cereb Blood Flow Met 12:448-455.

78. Choi IY, Lee SP, Kim SG, Gruetter R. 2001. In vivo measurements of brain glucose transport using the reversible Michaelis-Menten model and simultaneous measurements of cerebral blood flow changes during hypoglycemia. J Cereb Blood Flow Met 21:653663.

79. Criego AB, Tkac I, Kumar A, Thomas W, Gruetter R, Seaquist ER. 2005. Brain glucose concentrations in patients with type 1 diabetes and hypoglycemia unawareness. $J$ Neurosci Res 79:42-47.

80. Gruetter R, Rothman DL, Novotny EJ, Shulman GI, Prichard JW, Shulman RG. 1992. Detection and assignment of the glucose signal in ${ }^{\mathrm{I}} \mathrm{H}$ NMR spectra of the human brain. Magn Reson Med 26:183-188.

81. Choi IY, Lei H, Gruetter R. 2002. Effect of deep pentobarbital anesthesia on neurotransmitter metabolism in vivo: on the correlation of total glucose consumption with glutamatergic action. J Cereb Blood Flow Met 22:1343-1351.

82. de Graaf RA, Pan JW, Telang F, Lee JH, Brown P, Novotny EJ, Hetherington HP, Rothman DL. 2001. Differentiation of glucose transport in human brain gray and white matter. J Cereb Blood Flow Met 21:483-492.

83. Seaquist ER, Damberg GS, Tkac I, Gruetter R. 2001. The effect of insulin on in vivo cerebral glucose concentrations and rates of glucose transport/metabolism in humans. Diabetes 50:2203-2209.

84. Pfeuffer J, Tkac I, Gruetter R. 2000. Extracellular-intracellular distribution of glucose and lactate in the rat brain assessed noninvasively by diffusion-weighted ${ }^{1} \mathrm{H}$ nuclear magnetic resonance spectroscopy in vivo. J Cereb Blood Flow Met 20:736-746.

85. Gjedde A, Diemer NH. 1983. Autoradiographic determination of regional brain glucose content. J Cereb Blood Flow Met 3:303-310.

86. Holden JE, Mori K, Dienel GA, Cruz NF, Nelson T, Sokoloff L. 1991. Modeling the dependence of hexose distribution volumes in brain on plasma glucose concentration: implications for estimation of the local 2-deoxyglucose lumped constant. $J$ Cereb Blood Flow Met 11:171-182.

87. Lund-Andersen H. 1979. Transport of glucose from blood to brain. Physiol Rev 59:305-352.

88. Hasselbalch SG, Knudsen GM, Holm S, Hageman LP, Capaldo B, Paulson OB. 1996. Transport of D-glucose and 2-fluorodeoxyglucose across the blood-brain barrier in humans. J Cereb Blood Flow Met 16:659-666.

89. Gruetter R, Novotny EJ, Boulware SD, Rothman DL, Shulman RG. 1996. 'H NMR studies of glucose transport in the human brain. J Cereb Blood Flow Met 16:427-438.

90. Dienel GA, Cruz NF, Adachi K, Sokoloff L, Holden JE. 1997. Determination of local brain glucose level with $\left[{ }^{14} \mathrm{C}\right]$ methylglucose: effects of glucose supply and demand. $\mathrm{Am}$ $J$ Physiol 273:E839-849.

91. Cunningham VJ, Hargreaves RJ, Pelling D, Moorhouse SR. 1986. Regional bloodbrain glucose transfer in the rat: a novel double-membrane kinetic analysis. $J$ Cereb Blood Flow Met 6:305-314.

92. Gruetter R. 2003. Glycogen: the forgotten cerebral energy store. J Neurosci Res 74:179-183. 
93. Choi IY, Seaquist ER, Gruetter R. 2003. Effect of hypoglycemia on brain glycogen metabolism in vivo. $J$ Neurosci Res 72:25-32.

94. Lowry O, Passonneau J, Hasselberger F, Schulz D. 1964. Effect of ischemia on known substrates and cofactors of the glycolytic pathway in brain. $J$ Biol Chem 239:18-30.

95. Swanson RA, Sagar SM, Sharp FR. 1989. Regional brain glycogen stores and metabolism during complete global ischaemia. Neurol Res 11:24-28.

96. Cruz NF, Dienel GA. 2002. High glycogen levels in brains of rats with minimal environmental stimuli: implications for metabolic contributions of working astrocytes. $J$ Cereb Blood Flow Met 22:1476-1489.

97. Kong J, Shepel PN, Holden CP, Mackiewicz M, Pack Al, Geiger JD. 2002. Brain glycogen decreases with increased periods of wakefulness: implications for homeostatic drive to sleep. $J$ Neurosci 22:5581-5587.

98. Choi IY, Gruetter R. 2003. In vivo ${ }^{13} \mathrm{C}$ NMR assessment of brain glycogen concentration and turnover in the awake rat. Neurochem Int 43:317-322.

99. Sorg O, Magistretti PJ. 1992. Vasoactive intestinal peptide and noradrenaline exert long-term control on glycogen levels in astrocytes: blockade by protein synthesis inhibition. J Neurosci 12:4923-4931.

100. Fox PT, Raichle ME, Mintun MA, Dence C. 1988. Nonoxidative glucose consumption during focal physiologic neural activity. Science 241:462-464.

101. Prichard J, Rothman D, Novotny E, Petroff O, Kuwabara T, Avison M, Howseman A, Hanstock C, Shulman R. 1991. Lactate rise detected by ${ }^{\prime} \mathrm{H}$ NMR in human visual cortex during physiologic stimulation. Proc Natl Acad Sci USA 88:5829-5831.

102. Sappey-Marinier D, Calabrese G, Fein G, Hugg JW, Biggins C, Weiner MW. 1992. Effect of photic stimulation on human visual cortex lactate and phosphates using ${ }^{1} \mathrm{H}$ and ${ }^{31} \mathrm{P}$ magnetic resonance spectroscopy. J Cereb Blood Flow Met 12:584-592.

103. Merboldt KD, Bruhn H, Hanicke W, Michaelis T, Frahm J. 1992. Decrease of glucose in the human visual cortex during photic stimulation. Magn Reson Med 25:187-194.

104. Frahm J, Kruger G, Merboldt KD, Kleinschmidt A. 1996. Dynamic uncoupling and recoupling of perfusion and oxidative metabolism during focal brain activation in man. Magn Reson Med 35:143-148.

105. Madsen PL, Cruz NF, Sokoloff L, Dienel GA. 1999. Cerebral oxygen/glucose ratio is low during sensory stimulation and rises above normal during recovery: excess glucose consumption during stimulation is not accounted for by lactate efflux from or accumulation in brain tissue. J Cereb Blood Flow Met 19:393-400.

106. Shulman RG, Hyder F, Rothman DL. 2001. Cerebral energetics and the glycogen shunt: neurochemical basis of functional imaging. Proc Natl Acad Sci USA 98:64176422.

107. Chen W, Zhu XH, Gruetter R, Seaquist ER, Adriany G, Ugurbil K. 2001. Study of tricarboxylic acid cycle flux changes in human visual cortex during hemifield visual stimulation using ${ }^{1} \mathrm{H}-\left[{ }^{13} \mathrm{C}\right]$ MRS and fMRI. Magn Reson Med 45:349-355.

108. Ogawa S, Menon RS, Kim SG, Ugurbil K. 1998. On the characteristics of functional magnetic resonance imaging of the brain. Ann Rev Biophys Biomol Struct 27:447-474.

109. Berkich DA, Xu Y, LaNoue KF, Gruetter R, Hutson SM. 2005. Evaluation of brain mitochondrial glutamate and alpha-ketoglutarate transport under physiologic conditions. J Neurosci Res 79:106-113.

110. Henry PG, Lebon V, Vaufrey F, Brouillet E, Hantraye P, Bloch G. 2002. Decreased TCA cycle rate in the rat brain after acute 3-NP treatment measured by in vivo ${ }^{~} \mathrm{H}$ $\left[{ }^{13} \mathrm{C}\right]$ NMR spectroscopy. $J$ Neurochem 82:857-866. 
111. Yu X, White LT, Alpert NM, Lewandowski ED. 1996. Subcellular metabolite transport and carbon isotope kinetics in the intramyocardial glutamate pool. Biochemistry 35:6963-6968.

112. Jeffrey FM, Reshetov A, Storey CJ, Carvalho RA, Sherry AD, Malloy CR. 1999. Use of a single ${ }^{13} \mathrm{C}$ NMR resonance of glutamate for measuring oxygen consumption in tissue. Am J Physiol 277:E1111-1121.

113. Jucker BM, Ren J, Dufour S, Cao X, Previs SF, Cadman KS, Shulman GI. 2000. ${ }^{13} \mathrm{C} /{ }^{31} \mathrm{P}$ NMR assessment of mitochondrial energy coupling in skeletal muscle of awake fed and fasted rats: relationship with uncoupling protein 3 expression. $J$ Biol Chem 275:39279-39286.

114. LaNoue KF, Tischler ME. 1974. Electrogenic characteristics of the mitochondrial glutamate-aspartate antiporter. J Biol Chem 249:7522-7528.

115. Chatham JC, Forder JR, Glickson JD, Chance EM. 1995. Calculation of absolute metabolic flux and the elucidation of the pathways of glutamate labeling in perfused rat heart by ${ }^{13} \mathrm{C}$ NMR spectroscopy and nonlinear least squares analysis. $J$ Biol Chem 270:7999-8008.

116. Gruetter R. 2002. In vivo ${ }^{13} \mathrm{C}$ NMR studies of compartmentalized cerebral carbohydrate metabolism. Neurochem Int 41:143-154.

117. Oz G, Berkich DA, Henry PG, Xu Y, LaNoue K, Hutson SM, Gruetter R. 2004. Neuroglial metabolism in the awake rat brain: $\mathrm{CO} 2$ fixation increases with brain activity. $J$ Neurosci 24:11273-11279.

118. Yudkoff M, Nissim I, Daikhin Y, Lin Z, Nelson D, Pleasure D, Erecinska M. 1993. Brain glutamate metabolism: neuronal-astroglial relationships. Dev Neurosci 15:343350 .

119. Schousboe A, Westergaard N, Hertz L. 1993. Neuronal-astrocytic interactions in glutamate metabolism. Biochem Soc Trans 21:49-53.

120. McKenna MC, Stevenson JH, Huang X, Hopkins IB. 2000. Differential distribution of the enzymes glutamate dehydrogenase and aspartate aminotransferase in cortical synaptic mitochondria contributes to metabolic compartmentation in cortical synaptic terminals. Neurochem Int 37:229-241.

121. Daikhin Y, Yudkoff M. 2000. Compartmentation of brain glutamate metabolism in neurons and glia. J Nutr 130:1026S-1031S.

122. Schousboe A, Westergaard N, Sonnewald U, Petersen SB, Huang R, Peng L, Hertz L. 1993. Glutamate and glutamine metabolism and compartmentation in astrocytes. Dev Neurosci 15:359-366.

123. Nicklas WJ, Zeevalk G, Hyndman A. 1987. Interactions between neurons and glia in glutamate/glutamine compartmentation. Biochem Soc Trans 15:208-210.

124. Eriksson G, Peterson A, Iverfeldt K, Walum E. 1995. Sodium-dependent glutamate uptake as an activator of oxidative metabolism in primary astrocyte cultures from newborn rat. Glia 15:152-156.

125. Magistretti P, Pellerin L. 1996. Cellular mechanisms of brain energy metabolism: relevance to functional brain imaging and to neurodegenerative disorders. Ann $N Y$ Acad Sci 777:380-387.

126. Silver IA, Erecinska M. 1997. Energetic demands of the $\mathrm{Na}+\mathrm{K}+$ ATPase in mammalian astrocytes. Glia 21:35-45.

127. Tsacopoulos M, Magistretti P. 1996. Metabolic coupling between glia and neurons. $J$ Neurosci 16:877-885. 
128. Magistretti PJ, Pellerin L. 1999. Cellular mechanisms of brain energy metabolism and their relevance to functional brain imaging. Phil Trans Roy Soc London B (Biol Sci) 354:1155-1163.

129. Magistretti PJ, Sorg O, Yu N, Martin JL, Pellerin L. 1993. Neurotransmitters regulate energy metabolism in astrocytes: implications for the metabolic trafficking between neural cells. Dev Neurosci 15:306-312.

130. Bergles DE, Dzubay JA, Jahr CE. 1997. Glutamate transporter currents in bergmann glial cells follow the time course of extrasynaptic glutamate. Proc Natl Acad Sci USA 94:14821-14825.

131. Sibson NR, Dhankhar A, Mason GF, Rothman DL, Behar KL, Shulman RG. 1998. Stoichiometric coupling of brain glucose metabolism and glutamatergic neuronal activity. Proc Natl Acad Sci USA 95:316-321.

132. Attwell D, Laughlin SB. 2001. An energy budget for signaling in the grey matter of the brain. J Cereb Blood Flow Met 21:1133-1145.

133. Siegel GJ, Agranoff BW. 1999. Basic neurochemistry: molecular, cellular and medical aspects. Philadelphia: Lippincott-Raven Publishers.

134. Rothman DL, Sibson NR, Hyder F, Shen J, Behar KL, Shulman RG. 1999. In vivo nuclear magnetic resonance spectroscopy studies of the relationship between the glutamate-glutamine neurotransmitter cycle and functional neuroenergetics. Phil Trans Roy Soc London B (Biol Sci) 354:1165-1177

135. Lapidot A, Gopher A. 1994. Cerebral metabolic compartmentation: estimation of glucose flux via pyruvate carboxylase/pyruvate dehydrogenase by ${ }^{13} \mathrm{C} N \mathrm{NR}$ isotopomer analysis of D-[U- $\left.{ }^{13} \mathrm{C}\right]$ glucose metabolites. J Biol Chem 269:27198-27208.

136. Merle M, Bouzier-Sore AK, Canioni P. 2002. Time-dependence of the contribution of pyruvate carboxylase versus pyruvate dehydrogenase to rat brain glutamine labelling from $\left[1-{ }^{13} \mathrm{C}\right]$ glucose metabolism. $J$ Neurochem $82: 47-57$.

137. Berl S, Nicklas WJ, Clarke DD. 1970. Compartmentation of citric acid cycle metabolism in brain: labelling of glutamate, glutamine, aspartate and gaba by several radioactive tracer metabolites. J Neurochem 17:1009-1015.

138. Waniewski RA, Martin DL. 1998. Preferential utilization of acetate by astrocytes is attributable to transport. J Neurosci 18:5225-5233.

139. Dienel GA, Liu K, Popp D, Cruz NF. 1999. Enhanced acetate and glucose utilization during graded photic stimulation: neuronal-glial interactions in vivo. Ann NY Acad Sci 893:279-281.

140. Cruz-Aguado R, Francis-Turner L, Diaz CM, Antunez I. 2000. Quinolinic acid lesion induces changes in rat striatal glutathione metabolism. Neurochem Int 37:53-60.

141. Terpstra M, Gruetter R. 2004. ${ }^{1}$ H NMR detection of vitamin $C$ in human brain in vivo. Magn Reson Med 51:225-229. 